\title{
EXPONENTIAL STABILIZATION OF NONLINEAR DRIFTLESS SYSTEMS WITH ROBUSTNESS TO UNMODELED DYNAMICS *
}

\author{
Pascal Morin $^{1}$ and Claude Samson ${ }^{1}$
}

\begin{abstract}
Exponential stabilization of nonlinear driftless affine control systems is addressed with the concern of achieving robustness with respect to imperfect knowledge of the system's control vector fields. In order to satisfy this robustness requirement, and inspired by Bennani and Rouchon [1] where the same issue was first addressed, we consider a control strategy which consists in applying periodically updated open-loop controls that are continuous with respect to state initial conditions. These controllers are more precisely described as continuous time-periodic feedbacks associated with a specific dynamic extension of the original system. Sufficient conditions which, if they are satisfied by the control law, ensure that the control is a robust exponential stabilizer for the extended system are given. Explicit and simple control expressions which satisfy these conditions in the case of $n$ dimensional chained systems are proposed. A constructive algorithm for the design of such control laws, which applies to any (sufficiently regular) driftless control system, is described.
\end{abstract}

Résumé. Pour les systèmes de commande sans dérive, le problème de la stabilisation exponentielle est considéré, avec pour principal objectif l'obtention de commandes robustes par rapport aux erreurs de modélisation sur les champs de commande. Motivés par Bennani et Rouchon [1], où ce problème de robustesse a déjà été étudié, nous considérons des lois de commande en boucle ouverte, réinitialisées périodiquement, et dépendant continument de l'état initial. Ces lois de commande peuvent aussi être vues comme des retours d'état instationnaires continus définis à partir d'une extension dynamique particulière du système de départ. Nous énonçons des conditions suffisantes portant sur ces retours d'état pour qu'ils stabilisent de façon robuste le système étendu. Nous proposons ensuite des lois de commande explicites et simples qui satisfont ces conditions pour un système chaîné de dimension quelconque. Enfin, nous décrivons un algorithme de synthèse de lois de commande robustes, applicable à tout système régulier localement commandable.

AMS Subject Classification. 93D09, 93D15, 93D20.

Received July 31, 1998. Revised December 3, 1998.

Keywords and phrases: Nonlinear system, asymptotic stabilization, robust control, Chen-Fliess series.

* This work was partially supported by the GDR "Automatique".

${ }^{1}$ INRIA, 2004 route des Lucioles, 06902 Sophia-Antipolis Cedex, France; e-mail: first-name.last-name@inria.fr

(c) EDP Sciences, SMAI 1999 


\section{INTRODUCTION}

We consider an analytic driftless system on $\mathbb{R}^{n}$

$$
\left(S_{0}\right): \dot{x}=\sum_{i=1}^{m} f_{i}(x) u_{i},
$$

locally controllable around the origin, i.e.

$$
\operatorname{Span}\left\{f(0): f \in \operatorname{Lie}\left(f_{1}, \ldots, f_{m}\right)\right\}=\mathbb{R}^{n},
$$

and address the problem of constructing explicit feedback laws which (locally) exponentially stabilize, in some sense specified later, the origin $x=0$ of the controlled system. A further requirement is that these feedbacks should also be exponential stabilizers for any "perturbed" system in the form

$$
\left(S_{\epsilon}\right): \dot{x}=\sum_{i=1}^{m}\left(f_{i}(x)+h_{i}(\epsilon, x)\right) u_{i},
$$

with $h_{i}$ analytic in $\mathbb{R} \times \mathbb{R}^{n}$ and $h_{i}(0, x)=0$, when $|\epsilon|$ is small enough. In other words, given a nominal control system $\left(S_{0}\right)$, we would like to find nominal feedback controls, derived on the basis of this nominal system, that preserve the property of exponential stability when they are applied to "neighboring" systems $\left(S_{\epsilon}\right)$. It is of course assumed that the tangent linear approximation of $\left(S_{0}\right)$ at $(x=0, u=0)$ is not stabilizable, since otherwise the problem is simply solved by using an adequate linear feedback $u(x)=K x$. This assumption implies in particular that the rank of the matrix formed by the column-vectors $f_{i}(0), 1 \leq i \leq m$, is smaller than $n$.

In this context, the term $\sum_{i=1}^{m} h_{i}(\epsilon, x) u_{i}$ represents a class of unmodeled dynamics with respect to which the stabilizing nominal feedback must be robust. As pointed out in [16], such unmodeled dynamics may for example arise in practice, when dealing with nonholonomic wheeled vehicles, because of uncertainties upon the geometrical features of the vehicle. The present study is in fact essentially motivated by this robustness requirement. Indeed, explicit "homogeneous" exponential (time-varying) stabilizers $u(x, t)$ for systems $\left(S_{0}\right)$ have been derived in various previous studies (see $[13,15]$, for example). However, as demonstrated recently in [12], none of these controls solves the robustness problem stated above in the sense that there always exists some $h_{i}(\epsilon,$.$) for which the origin of the associated controlled system is not stable when \epsilon \neq 0$. Note that this negative result does not contradict the fact that such controllers are robust against less general unmodeled dynamics, as this is ensured by the existence of a Lyapunov function for the controlled nominal system. It just emphasizes the fact that, contrary to the case of linear control systems stabilized via the use of linear feedbacks, the existence of a Lyapunov function for the controlled system is not sufficient to ensure the type of robustness considered here. This negative result also strongly suggests (although this remains to be rigorously established) that no continuous feedback $u(x, t)$, not necessarily homogeneous, can be a robust exponential stabilizer. However, it does not imply that the problem cannot be handled via an adequate dynamic extension of the original nominal system. Proving the existence, or non-existence, of solutions of this type could thus be a subject for future studies. As a matter of fact, and as explained below, the present study may already be seen as a step in this direction.

Feedbacks which are continuous with respect to the state do not represent the only "reasonable" possibility in order to achieve the desired robustness result. For instance, besides feedbacks which are discontinuous at the origin, as proposed by several authors in the past (see $[3,8]$, for example) and for which robustness issues have seldom been addressed so far, a possibility consists in considering hybrid open-loop/feedback control strategies such as open-loop controls which are periodically updated from the measurement $x(k T), k \in \mathbb{N}$, of the state at discrete time-instants. Such a control has features shared by classical piecewise-constant discretetime feedbacks, but unlike these (and for the purpose of asymptotic stabilization) the control value between 
two sampling time-instants cannot, in the present case, be constant because this would contradict the known non-existence (resulting from the violation of Brockett's condition [2]) of asymptotically stabilizing continuous pure-state feedbacks $u(x)$. The idea of using this type of control for the purpose of stabilizing the class of driftless systems considered here is not new. This possibility has sometimes been presented as an extension of solutions obtained when addressing the open-loop steering problem, i.e. the problem of finding an open-loop control which steers the system from an initial state to another desired one (see [14,18], for example). Hybrid continuous/discrete time exponential stabilizers for chained systems, which do not specifically rely on open-loop steering control, have also been proposed in [21]. However, [1] is to our knowledge the only study where the robustness problem stated above has been formulated in a similar fashion and where it has been shown that this problem can be solved by using a hybrid open-loop/feedback control. More precisely, the above reference i) proposes a methodology for constructing a robust control solution in the specific case (of practical interest) when the nominal system $\left(S_{0}\right)$ is a 2-input $n$-dimensional chained system, ii) describes sufficient conditions for the $n$-dimensional case which, if they are met by the control expression, ensures robust stabilization, and iii) provides an explicit control expression in the 4-dimensional case (the dimension 4 having been chosen merely to show that the approach remains tractable for dimensions larger than 3). In fact, although this is not specified in the above reference, the proposed control does not "strictly" ensure asymptotic stability, in the usual sense of Lyapunov, of the origin of the perturbed systems $\left(S_{\epsilon}\right)$.

In order to be more specific about the latter point, and also clarify the meaning of "periodically" updated open-loop control applied to a time-continuous system $\dot{x}=f(x, u)$ ", it is useful to introduce the following extended control system:

$$
\left\{\begin{array}{l}
\dot{x}=f(x, u) \\
\dot{y}=\left(\sum_{k \in \mathbb{N}} \delta_{k T}\right)\left(x-y_{-\alpha}\right), \quad 0<\alpha<T,
\end{array}\right.
$$

with $T$ denoting the updating time-period of the control part which depends upon $y, \delta_{k T}$ the classical dirac impulse at the time-instant $k T$, and $y_{-\alpha}$ the delay operator such that $y_{-\alpha}(t)=y(t-\alpha)$. Given a continuous feedback control $u(x, y, t)$, an initial condition $\left(x\left(t_{0}\right), y\left(t_{0}\right)\right)$ to the controlled extended system is defined by i) choosing a point $\left(x_{0}, y_{0}\right) \in \mathbb{R}^{n} \times \mathbb{R}^{n}$, and ii) setting $x\left(t_{0}\right)=x_{0}, y\left(t_{0}\right)=y_{0}$ if $t_{0}$ is not a multiple of $T$, and $y\left(t_{0}\right)=x_{0}$ if $t_{0}=k T$. The introduction of the extra equation in $y$ just indicates that $y(t)$ is constant and equal to $x(k T)$ on the time-interval $[k T,(k+1) T)$. Therefore, any control the expression of which, on the time-interval $[k T,(k+1) T)$, is a function of $x(k T)$ and $t$, may just be interpreted as a feedback control $u(y, t)$ for the corresponding extended system. From now on, we will adopt this point of view whenever referring to this type of control. As commonly done elsewhere, we will also say that a feedback control $u(x, y, t)$ is a (uniform) exponential stabilizer for the extended system (4) if there exist an open set $U \in \mathbb{R}^{n} \times \mathbb{R}^{n}$ containing the point $(0,0)$, a positive real number $\gamma$, and a function $\beta$ of class $\mathcal{K}$ such that:

$$
|(x(t), y(t))| \leq \beta\left(\left|\left(x\left(t_{0}\right), y\left(t_{0}\right)\right)\right|\right) \exp \left(-\gamma\left(t-t_{0}\right)\right) \forall t \geq t_{0} \geq 0 ; \forall\left(x\left(t_{0}\right), y\left(t_{0}\right)\right) \in U
$$

with $(x(t), y(t))$ denoting any solution of the controlled system. Note that the satisfaction of (5) does not imply that the control is an exponential stabilizer for the original system (while the converse is true). It is thus a slightly weaker property. However, except for asking finite-time convergence to zero, it is nearly the best that can be obtained when using a control which depends continuously upon $y$, knowing that such a control cannot by construction asymptotically stabilize the original system. For instance $x(t)$ may well cross zero at a time-instant which is not a multiple of $T$, without stopping there. Note that a similar impossibility holds when the feedback control depends on the integral of the state $x$ : asymptotic stability can only be established for an extended state which contains the integral of $x$. This has not prevented linear PID controllers from being popular and widely employed in practice. 
Let us now come back to the control strategy studied in [1] and interpret it in view of the above definitions. It yields continuous time-periodic feedbacks $u(y, t)$ (i.e. such that $u\left(y, t+T^{\prime}\right)=u(y, t)$ for some $\left.T^{\prime}>0\right)$, the time-periods of which are equal to the updating period $T$ (i.e. $T^{\prime}=T$ ). In our opinion, the importance of the contribution in [1] comes from that it convincingly demonstrates the possibility of achieving robust (with respect to unmodeled dynamics, as defined earlier) exponential stabilization (stability being now taken in the strict sense of Lyapunov) of an extended control system

$$
\left(\bar{S}_{0}\right):\left\{\begin{array}{l}
\dot{x}=\sum_{i=1}^{m} f_{i}(x) u_{i} \\
\dot{y}=\left(\sum_{k \in \mathbb{N}} \delta_{k T}\right)\left(x-y_{-\alpha}\right), \quad 0<\alpha<T
\end{array}\right.
$$

via the use of a continuous time-periodic feedback $u(y, t)$.

In the present paper, the exploration of this possibility is carried further on. The first result provides a sufficient condition under which a time-periodic continuous feedback controller $u(y, t)$ i) exponentially stabilizes the origin of a system (6), and also ii) exponentially stabilizes the origin of any neighboring system

$$
\left(\bar{S}_{\epsilon}\right):\left\{\begin{array}{l}
\dot{x}=\sum_{i=1}^{m}\left(f_{i}(x)+h_{i}(\epsilon, x)\right) u_{i} \\
\dot{y}=\left(\sum_{k \in \mathbb{N}} \delta_{k T}\right)\left(x-y_{-\alpha}\right) 0<\alpha<T
\end{array}\right.
$$

provided that $|\epsilon|$ is small enough. Then, on the basis of this result, we propose a systematic and complete control design procedure which only involves a finite number of algebraic operations. Following this design procedure thus yields entirely explicit feedback laws. The procedure is itself adapted from existing time-periodic open-loop control design techniques which have been proposed in [11,24] (see also [10] for an early but complete survey of such techniques) for driftless control systems affine in the control. Although the implementation of the resulting algorithm is somewhat involved in the general case, we show that simple control expressions can be obtained for specific classes of systems, as illustrated in the case where the original system $\left(S_{0}\right)$ is in the chained form. Also, with respect to the solution given in [1] for the 4-dimensional chained system, the single control expression proposed here encompasses all dimensions with no extra work needed.

The paper is organized as follows. The possiblity of achieving asymptotic stabilization with robustness to errors in the modeling of the system's control vector fields, via the use of a hybrid time-periodic feedback control, is illustrated on a simple example in Section 2. For a general driftless affine control system, sufficient conditions for exponential and robust stabilization are then stated in Section 3. The design of time-periodic feedbacks $u(y, t)$ which satisfy these conditions is carried out in Section 4. A general control design algorithm, which applies to any locally controllable driftless system, is first described in Subsection 4.1. The possibility of synthesizing robust controllers from an homogeneous approximation of the control system is treated in Subsection 4.2. This yields to imposing an extra condition on the control law. For $n$-dimensional chained systems, robust feedback controls the expression of which is simpler (when $n>3$ ) than these obtained by application of the general algorithm are proposed in Subsection 4.3. A few final remarks are given in Section 5. The proofs of the main results are reported in the Appendix.

The following notation is used.

- The identity function on $\mathbb{R}^{n}$ is denoted $i d,|$.$| is the Euclidean norm, and |\cdot|_{P}$ is the norm induced by a positive definite matrix $P$.

- The transpose of a row-vector $\left(x_{1}, \ldots, x_{n}\right)$ is denoted as $\left(x_{1}, \ldots, x_{n}\right)^{\prime}$. 
- For any vector field $X$ and smooth function $f$ on $\mathbb{R}^{n}, X f$ denotes the Lie derivative of $f$ along the vector field $X$. When $f=\left(f_{1}, \ldots, f_{n}\right)^{\prime}$ is a smooth map from $\mathbb{R}^{n}$ to itself, $X f$ denotes the map $\left(X f_{1}, \ldots, X f_{n}\right)^{\prime}$.

- A square matrix $A$ is called discrete-stable if all its eigenvalues are strictly inside the complex unit circle.

- Given a continuous functions $g$, defined on some neighborhood of the origin in $\mathbb{R}^{n}$, we denote $o(g)$ (resp. $O(g)$ ) any function or map such that $\frac{|O(g)(x)|}{|g(x)|} \longrightarrow 0$ as $|x| \longrightarrow 0$ (resp. such that $\frac{|O(g)(x)|}{|g(x)|} \leq K$ in some neighborhood of the origin). When $g=|$.$| , we write o(x)($ resp. $O(x))$ instead of $o(g)(x)($ resp. $O(g)(x))$.

\section{A SIMPLE EXAMPLE}

This example is given so as to get acquainted with the feedback control solutions which are developped subsequently. Let us consider the following three-dimensional chained system.

$$
\left(S_{0}\right):\left\{\begin{array}{l}
\dot{x}_{1}=u_{1} \\
\dot{x}_{2}=u_{2} \\
\dot{x}_{3}=u_{1} x_{2} .
\end{array}\right.
$$

While being the simplest control system of relevance to the present study, it is commonly used to model the kinematics of unicycle-type nonholonomic vehicles. It is also the system which, starting with [20], has triggered all recent developments about continuous time-varying stabilizers.

Let us consider a modeling error $h_{1}=\epsilon \frac{\partial}{\partial x_{3}}$ associated with the first control vector field, so that the corresponding "perturbed" control system is

$$
\left(S_{\epsilon}\right):\left\{\begin{array}{l}
\dot{x}_{1}=u_{1} \\
\dot{x}_{2}=u_{2} \\
\dot{x}_{3}=u_{1} x_{2}+\epsilon u_{1} .
\end{array}\right.
$$

We have chosen this specific modeling error because it is a typical example of a term which "destabilizes" the origin of the system, whatever the homogeneous continuous time-periodic exponential stabilizer derived for the nominal system $\left(S_{0}\right)$. Instead of such a continuous feedback, let us rather consider the hybrid time-periodic feedback defined by

$$
\begin{aligned}
& u_{1}(x(k T), t)=\frac{1}{T}\left[-x_{1}(k T)+2 \pi\left|x_{3}(k T)\right|^{1 / 2} \operatorname{sign}\left(x_{3}(k T)\right) \sin \left(\frac{2 \pi t}{T}\right)\right] \\
& u_{2}(x(k T), t)=\frac{1}{T}\left[-x_{2}(k T)-2\left|x_{3}(k T)\right|^{1 / 2} \cos \left(\frac{2 \pi t}{T}\right)\right]
\end{aligned}
$$

for $t \in[k T,(k+1) T), k \in \mathbb{N}$, and with $T \in \mathbb{R}^{+}$denoting the updating time-period of the control. 
It is in this case quite simple to integrate the equations of the controlled perturbed system. One obtains in particular

$$
\begin{aligned}
x_{1}((k+1) T)= & x_{1}(k T)+\int_{k T}^{(k+1) T} u_{1}(x(k T), s) d s=0 \\
x_{2}((k+1) T)= & x_{2}(k T)+\int_{k T}^{(k+1) T} u_{2}(x(k T), s) d s=0 \\
x_{3}((k+1) T)= & x_{3}(k T)+\int_{k T}^{(k+1) T} x_{2}(s) u_{1}(x(k T), s)+\epsilon u_{1}(x(k T), s) d s \\
= & x_{3}(k T)+\int_{k T}^{(k+1) T} u_{1}(x(k T), s)\left[\epsilon+x_{2}(k T)+\int_{k T}^{s} u_{2}(x(k T), \tau) d \tau\right] d s \\
= & x_{3}(k T)+\left(\epsilon+x_{2}(k T)\right) \int_{k T}^{s} u_{1}(x(k T), s) d s \\
& +\int_{k T}^{(k+1) T} u_{1}(x(k T), s) \int_{k T}^{s} u_{2}(x(k T), \tau) d \tau d s \\
= & -\left(\epsilon+x_{2}(k T)\right) x_{1}(k T)+\frac{1}{2} x_{1}(k T) x_{2}(k T)+x_{2}(k T)\left|x_{3}(k T)\right| \operatorname{sign}\left(x_{3}(k T)\right) .
\end{aligned}
$$

One deduces from (11) that

$$
\begin{array}{ll}
x_{1}(k T)=x_{2}(k T)=0 & \forall k \geq 1 \\
x_{3}(k T)=0 & \forall k \geq 2
\end{array}
$$

and $x(t)=0, \forall t \geq 2 T$ (since the control law vanishes at $x=0$ ). Therefore, all trajectories of the perturbed controlled system globally converge to zero in finite time whatever the size of $\epsilon$.

This example is particular in the sense that we shall not try to guarantee, for every system, finite-time convergence of the trajectories to zero. In the general case, the objective will be to obtain for the controlled system a relation in the form

$$
x((k+1) T)=A x(k T)+\epsilon O(x)(k T)+o(x)(k T)
$$

with $A$ a discrete-stable matrix. Note that this relation is satisfied for the above example with $A=0$.

\section{SUFFICIENT CONDITIONS FOR EXPONENTIAL AND ROBUST STABILIZATION}

Prior to stating the main result of this section, we review some properties of Chen-Fliess series that will be used in the sequel. The exposition is based on $[6,25]$, and limited here to driftless systems.

A $m$-valued multi-index $I$ is a vector $I=\left(i_{1}, \ldots, i_{k}\right)$ with $k$ denoting a strictly positive integer, and $i_{1}, \ldots, i_{k}$ integers taken in the set $\{1, \ldots, m\}$. We denote the length of $I$ as $|I|$, i.e. $I=\left(i_{1}, \ldots, i_{k}\right) \Longrightarrow|I|=k$.

Given piecewise continuous functions $u_{1}, \ldots, u_{m}$ defined on some time-interval $[0, T]$, and a $m$-valued multi-index $I=\left(i_{1}, \ldots, i_{k}\right)$, we define

$$
\int_{0}^{t} u_{I}=\int_{0}^{t} \int_{0}^{t_{k}} \cdots \int_{0}^{t_{2}} u_{i_{k}}\left(t_{k}\right) u_{i_{k-1}}\left(t_{k-1}\right) \cdots u_{i_{1}}\left(t_{1}\right) d t_{1} \cdots d t_{k} \quad(t \in[0, T]) .
$$

Given smooth vector fields $f_{1}, \ldots, f_{m}$ on $\mathbb{R}^{n}$, and a $m$-valued multi-index $I=\left(i_{1}, \ldots, i_{k}\right)$, we define the $k$-th order differential operator $f_{I}: \mathcal{C}^{\infty}\left(\mathbb{R}^{n} ; \mathbb{R}\right) \longrightarrow \mathcal{C}^{\infty}\left(\mathbb{R}^{n} ; \mathbb{R}\right)$ by

$$
f_{I} g=f_{i_{1}} f_{i_{2}} \cdots f_{i_{k}} g
$$

The following proposition is a classical result (see e.g. [25] for the proof). 
Proposition 1. [25] Consider the analytic system $\left(S_{0}\right)$ and a compact set $K \subset \mathbb{R}^{n}$. There exists $\mu>0$ such that for $M, T \geq 0$ verifying

$$
M T \leq \mu,
$$

and for any control $u=\left(u_{1}, \ldots, u_{m}\right)$ piecewise continuous on $[0, T]$ and verifying

$$
|u(t)| \leq M, \quad \forall t \in[0, T]
$$

the solution $x($.$) of \left(S_{0}\right)$, with $x_{0} \triangleq x(0) \in K$, satisfies

$$
x(t)=x_{0}+\sum_{I}\left(f_{I} i d\right)\left(x_{0}\right) \int_{0}^{t} u_{I}, \quad \forall t \in[0, T] .
$$

Furthermore, the series in the right-hand side of (16) is uniformly absolutely convergent w.r.t. $t \in[0, T]$ and $x_{0} \in K$.

Note that the sum in the right-hand side of equality (16) can be developed as

$$
\sum_{k=1}^{\infty} \sum_{i_{1}, \ldots, i_{k}=1}^{m}\left(f_{i_{1}} \cdots f_{i_{k}} i d\right)\left(x_{0}\right) \int_{0}^{t} \int_{0}^{t_{k}} \cdots \int_{0}^{t_{2}} u_{i_{k}}\left(t_{k}\right) u_{i_{k-1}}\left(t_{k-1}\right) \cdots u_{i_{1}}\left(t_{1}\right) d t_{1} \cdots d t_{k} .
$$

Let us also remark that the condition (14), which relates the integration time-interval to the control size, is specific to driftless systems. For a system which contains a drift term, it is a priori not true that decreasing the size of the control inputs allows to increase the time-interval on which the expansion (16) is valid. The fact that this property holds for driftless systems can be viewed as a consequence of time-scaling invariance properties.

We are now ready to state sufficient conditions under which exponential stabilization robust to unmodeled dynamics is granted.

Theorem 1. Consider an analytic locally controllable system $\left(S_{0}\right)$, a neighborhood $U$ of the origin in $\mathbb{R}^{n}$, and a function $u \in \mathcal{C}^{0}\left(U \times \mathbb{R}^{+} ; \mathbb{R}^{m}\right)$ with $u(x,$.$) T-periodic for all x$. Assume that:

1. there exist $\alpha, K>0$ such that $|u(x, t)| \leq K|x|^{\alpha}$ for all $(x, t) \in U \times[0, T]$,

2. the solution $x($.$) of$

$$
\dot{x}=\sum_{i=1}^{m} f_{i}(x) u_{i}\left(x_{0}, t\right), \quad x(0)=x_{0} \in U
$$

satisfies $x(T)=A x_{0}+o\left(x_{0}\right)$ with $A$ a discrete-stable matrix,

3. for any multi-index $I$ of length $|I| \leq 1 / \alpha$ (this assumption is only needed when $\alpha<1$ ),

$$
\int_{0}^{T} u_{I}(x)=O(x)
$$

Then, given a family of perturbed systems $\left(S_{\epsilon}\right)$, there exists $\epsilon_{0}>0$ such that the origin of $\left(\bar{S}_{\epsilon}\right)$ controlled by $u(y, t)$ is locally exponentially stable for any $\epsilon \in\left(-\epsilon_{0}, \epsilon_{0}\right)$.

Remark: A natural question which arises at this point is whether a control feedback which verifies the assumptions of Theorem 1 can yield asymptotic stability of the origin of the system when an unmodeled (unknown) drift vector field, say $h_{0}(\epsilon, x)$ such that $h_{0}(0,)=.h_{0}(., 0)=0$, is added to the system. In the general case, when the linear approximation of the nominal system is not controllable, the answer to this question is 
negative. Robustness with respect to such a drift term is only obtained by imposing further restrictions upon the variation of $h_{0}(\epsilon, x)$ near $x=0$, as in the case of continuous homogeneous time-periodic feedbacks. For instance, it is not difficult to show that if the assumptions of Theorem 1 are verified, then there exists a positive number $\gamma(>1)$ such that the conclusion of the theorem holds when $h_{0}(\epsilon, x)$ is a $O\left(|x|^{\gamma}\right)$. This is however a rather weak statement, and refinement of this result could be the subject of a complementary study.

\section{Control Design}

This section addresses the problem of constructing explicit controllers that meet the conditions of Theorem 1. Such controllers have to be exponential stabilizers for the extended system $\left(\bar{S}_{0}\right)$. A general design algorithm is first proposed. It takes advantage of known techniques based on the use of oscillatory open-loop controls in order to achieve net motion in any direction of the state space. Unfortunately (and unavoidably), the procedure also inherits the complexity of the abovementioned techniques, itself directly related to the process of selecting the "right" frequencies which facilitate motion monitoring in the state space. Unsurprisingly, the selection of these frequencies gets all the more involved that controllability of the system relies on high-order Lie brackets of the control vector fields. The control design can be carried out from the expression of either the original system $\left(S_{0}\right)$ or any locally controllable homogeneous approximation of $\left(S_{0}\right)$. Indeed, working with an homogeneous approximation preserves the robustness of the feedback law provided that an extra condition is satisfied by the control law. This is shown after recalling basic definitions and facts about homogeneous systems. Working with homogeneous approximations is of interest in order to simplify the calculations involving the control vector fields (this is due to the nilpotent structure of homogeneous vector fields). These approximations may also exhibit structural properties useful to simplify the control design. This is illustrated on chained systems for which we propose simple robust controllers.

\subsection{A general control design procedure}

We present in this section a general algorithm to construct robust and exponential stabilizers for $\left(S_{0}\right)$. The algorithm uses previous results by Sussmann and Liu [24], and Liu [11]. It is also much related to the one developed in [15] for the construction of continuous time-periodic feedbacks $u(x, t)$ which exponentially stabilize the origin of a driftless system $\left(S_{0}\right)$, but present the shortcoming of not being endowed with the type of robustness here considered.

The presentation of the algorithm is organized as follows. First, we provide a compact and digest description of the control construction. Then, we give complementary explanations and comments, which may be useful to readers who are not familiar with every aspect involved in the construction. These explanations are also used in the subsequent stability and robustness analysis.

In order to make the exposition more rigorous, we first recall some notations from [26]. To the set of control vector fields $\left\{f_{1}, \ldots, f_{m}\right\}$ we associate a set of indeterminates $X=\left\{X_{1}, \ldots, X_{m}\right\}$. Brackets in $L(X)$, the free Lie algebra in the indeterminates $X_{1}, \ldots, X_{m}$, will be denoted with the letter $\mathcal{B}$. To any such bracket, one can associate a length and a set of indeterminates. For instance, $\mathcal{B}=\left[X_{1},\left[X_{2}, X_{1}\right]\right]$ has length three, and his set of indeterminates is $\left\{X_{1}, X_{2}, X_{1}\right\}$. To each element $A$ in $L(X)$, one can also associate an element in the control Lie algebra Lie $(f)$ by means of the evaluation operator Ev. More precisely, $E v(f)(A)$ is the vector field obtained by plugging in the $f_{j}$ 's for the $X_{j}$ 's in $A$. For instance, if $\mathcal{B}=\left[X_{1}, X_{2}\right]$, then $E v(f)(\mathcal{B})$ is the vector field $\left[f_{1}, f_{2}\right]$. Let us remark that if the control system $\left(S_{0}\right)$ were the only one under consideration, the concept of brackets in free Lie algebras would not be necessary. However, since the objective is to derive a single control expression which allows to asymptotically stabilize the origin of a whole family of systems $\left(S_{\epsilon}\right)$, it is understandably of interest and useful to pinpoint structural properties which are shared by all members of this family, independently of small differences in their respective control vector fields. Such properties are more easily expressed and analyzed in the framework of free Lie algebras. 


\section{Design algorithm}

Step 1. Determine $n$ vector fields $\tilde{f}_{j}(j=1, \ldots, n)$, obtained as Lie brackets of length $\ell(j)$ of the control vector fields $f_{i}$, and such that the matrix

$$
\tilde{F}(x) \triangleq\left(\tilde{f}_{1}(x), \ldots, \tilde{f}_{n}(x)\right)
$$

is nonsingular at $x=0$.

Step 2. Determine a matrix $G$ such that the matrix $\left(I_{n}+\tilde{F}(0) G\right)$ is discrete-stable (with $I_{n}$ denoting the n-dimensional identity matrix), and define the linear feedback

$$
a(x)=\frac{1}{T} G x .
$$

Step 3. By Step 1 , there exists, for each $j=1, \ldots, n$, a bracket $\mathcal{B}_{j}$ such that $\tilde{f}_{j}=E v(f)\left(\mathcal{B}_{j}\right)$. Partition the set $\left\{\mathcal{B}_{1}, \ldots, \mathcal{B}_{n}\right\}$ in homogeneous components $P_{1}, \ldots, P_{K}$, i.e.

i) All brackets in a homogeneous component $P_{k}$ have the same length $l(k)$, and the same set of indeterminates $\left\{X_{\tau_{1}^{k}}, \ldots, X_{\tau_{l(k)}^{k}}\right\}$

ii) Given two homogeneous components $P_{k}$ and $P_{k^{\prime}}\left(\right.$ with $\left.k \neq k^{\prime}\right)$, either $l(k) \neq l\left(k^{\prime}\right)$, or $\left\{X_{\tau_{1}^{k}}, \ldots, X_{\tau_{l(k)}^{k}}\right\} \neq$ $\left\{X_{\tau_{1}^{k^{\prime}}}, \ldots, X_{\tau_{l\left(k^{\prime}\right)}^{k^{\prime}}}\right\}$

Step 4. The last four steps can be conducted either in the control Lie algebra (c.l.a.) framework or in the framework of free Lie algebras (f.l.a.) ${ }^{1}$.

c.l.a.: For every $k=1, \ldots, K$, find permutations $\sigma_{1}, \ldots, \sigma_{\underline{C}(k)}$ in $\mathfrak{S}(l(k))$ such that the vector fields

$$
\left[f_{\tau_{\sigma(1)}^{k}},\left[f_{\tau_{\sigma(2)}^{k}},\left[\ldots, f_{\tau_{\sigma(l(k))}^{k}}\right] \ldots\right]\right] \quad\left(\sigma \in\left\{\sigma_{1}, \ldots, \sigma_{\underline{C}(k)}\right\}\right)
$$

form a basis of the linear sub-space (over $\mathbb{R}$ ) of $\operatorname{Lie}(f)$ spanned by the vector fields

$$
\left[f_{\tau_{\sigma(1)}^{k}},\left[f_{\tau_{\sigma(2)}^{k}},\left[\ldots, f_{\tau_{\sigma(l(k))}^{k}}\right] \ldots\right]\right] \quad(\sigma \in \mathfrak{S}(l(k))) .
$$

f.l.a.: For every $k=1, \ldots, K$, find permutations $\sigma_{1}, \ldots, \sigma_{\bar{C}(k)}$ in $\mathfrak{S}(l(k))$ such that the brackets

$$
\left[X_{\tau_{\sigma(1)}^{k}},\left[X_{\tau_{\sigma(2)}^{k}},\left[\ldots, X_{\tau_{\sigma(l(k))}^{k}}\right] \ldots\right]\right] \quad\left(\sigma \in\left\{\sigma_{1}, \ldots, \sigma_{\bar{C}(k)}\right\}\right)
$$

form a basis of the linear sub-space (over $\mathbb{R}$ ) of $L(X)$ spanned by the brackets

$$
\left[X_{\tau_{\sigma(1)}^{k}},\left[X_{\tau_{\sigma(2)}^{k}},\left[\ldots, X_{\tau_{\sigma(l(k))}^{k}}\right] \ldots\right]\right] \quad(\sigma \in \mathfrak{S}(l(k))) .
$$

\section{Step 5.}

c.l.a.: For every $k \in\{1, \ldots, K\}$ such that $l(k) \geq 2$, determine $C(k) \triangleq \underline{C}(k)$ Minimally Cancelling (MC) sets $\Omega^{k, c}=\left\{\omega_{1}^{k, c}, \ldots, \omega_{l(k)}^{k, c}\right\}$, with $c=1, \ldots, C(k)$, such that

\footnotetext{
${ }^{1}$ Respective advantages and drawbacks will be pointed out later.
} 
i) the family of sets $\left(\Omega^{k, c}\right)_{c=1, \ldots, C(k)}^{k=1, \ldots, K}$ is independent w.r.t. $\max _{k \in\{1, \ldots, K\}} l(k)$,

ii) all elements in these sets have a (positive) common divisor $\bar{\omega}\left(=\frac{2 \pi}{T}\right)$, i.e. $\frac{\omega_{i}^{k, c}}{\bar{\omega}} \in \mathbb{Z}, \forall(k, c, i)$,

iii) the $C(k)$ elements $g^{k, c}(c=1, \ldots, C(k))$ of $\operatorname{Lie}(f)$ defined by

$$
g^{k, c}=\sum_{\sigma \in \mathfrak{S}(l(k))} \frac{\left[f_{\tau_{\sigma(1)}^{k}},\left[f_{\tau_{\sigma(2)}^{k}},\left[\ldots, f_{\tau_{\sigma(l(k))}^{k}}\right] \ldots\right]\right]}{\omega_{\sigma(1)}^{k, c}\left(\omega_{\sigma(1)}^{k, c}+\omega_{\sigma(2)}^{k, c}\right) \cdots\left(\omega_{\sigma(1)}^{k, c}+\ldots+\omega_{\sigma(l(k)-1)}^{k, c}\right)}
$$

are independent (over $\mathbb{R}$ ).

For every $k \in\{1, \ldots, K\}$ such that $l(k)=1$, just set $\omega_{1}^{k, 1}=0$.

Each family of sets $\left\{\Omega^{k, c}\right\}_{c=1, \ldots, C(k)}$ is used to associate the following sine and cosine functions with $P_{k}$

$$
\alpha_{\tau_{i}^{k}}^{k, c}(t)= \begin{cases}\cos \omega_{i}^{k, c} t & (i=1) \\ \sin \omega_{i}^{k, c} t & (i=2, \ldots, l(k)) .\end{cases}
$$

f.l.a.: Same as above, with $C(k) \triangleq \bar{C}(k)$ instead of $\underline{C}(k)$, each $f_{i}$ replaced by $X_{i}$, and $L i e(f)$ replaced by $L(X)$.

\section{Step 6.}

c.l.a.: For each $k \in\{1, \ldots, K\}$ and $j$ such that $\mathcal{B}_{j} \in P_{k}$, determine coefficients $\mu_{j}^{k, c}(c=1, \ldots, C(k))$ such that

$$
\tilde{f}_{j}=\frac{(-1)^{l(k)-1}}{l(k) 2^{l(k)-1}} \sum_{c=1}^{C(k)} \mu_{j}^{k, c} g^{k, c}
$$

f.l.a.: Same as above, with $\tilde{f}_{j}$ replaced by $\mathcal{B}_{j}$.

Step 7.

c.l.a. and f.l.a.: For each $k \in\{1, \ldots, K\}$, determine $l(k) C(k)$ state dependent functions $v_{\tau_{i}^{k}}^{k, c}$ which are $O\left(|x|^{\frac{1}{l(k)}}\right)$, and such that

$$
\prod_{i=1}^{l(k)} v_{\tau_{i}^{k}}^{k, c}(x)=\sum_{j: \mathcal{B}_{j} \in P_{k}} \mu_{j}^{k, c} a_{j}(x)
$$

( $a_{j}$ is the $j$-th component of $a$ defined by (20)).

The following result concludes the description of the algorithm.

Theorem 2. Let

$$
u_{i}(x, t)=\left\{\begin{array}{cl}
\sum_{k=1}^{K} \sum_{c=1}^{C(k)} \sum_{p: \tau_{p}^{k}=i} \alpha_{\tau_{p}^{k}}^{k, c}(t) v_{\tau_{p}^{k}}^{k, c}(x) & \text { if } \exists(k, p): \tau_{p}^{k}=i \\
0 & \text { otherwise }
\end{array}\right.
$$

with $C(k)$ equal to $\underline{C}(k)$ in the c.l.a. case, and to $\bar{C}(k)$ in the f.l.a. case. Then,

i) in both cases, $u$ defined by (25) belongs to $\mathcal{C}^{0}\left(\mathbb{R}^{n} \times \mathbb{R}^{+} ; \mathbb{R}^{m}\right)$, and satisfies the three assumptions of Theorem 1, 
ii) in the f.l.a. case, local asymptotic stability of the origin of the perturbed system $\left(\bar{S}_{\epsilon}\right)$ is guaranted for any $\epsilon$ such that $I_{n}+\tilde{F}_{\epsilon}(0) G$ is discrete-stable, where $\tilde{F}_{\epsilon}$ denotes the matrix-valued function obtained from (19) by replacing each $\tilde{f}_{j}=E v(f)\left(\mathcal{B}_{j}\right)$ by $\tilde{f}_{j, \epsilon}=E v(f+h(\epsilon,)).\left(\mathcal{B}_{j}\right)$.

The above property $i$ ) points out the main advantage of working in the f.l.a. framework: asymptotic stability of the origin of the controlled perturbed system is simply equivalent to discrete-stability of the matrix $I_{n}+\tilde{F}_{\epsilon}(0) G$. This result is conceptually interesting because it is reminiscent of a classical robustness property obtained in the case of linear control systems.

We now review all steps of the construction so as to give a few complementary explanations and comments. At the same time, we show that each step can be carried out.

- Step 1. The existence of $n$ vector fields $\tilde{f}_{j}$ which satisfy the required condition is guaranteed by the local controllability of system $\left(S_{0}\right)$. In view of property ii) of Theorem 2, when this set of vector fields is not unique, the selection of this set has a clear influence on the domain of robustness of the resulting control.

- Step 2. The choice of $G$, as indicated in Step 2, can be interpreted in the following way. $G$ is calculated so that the linear feedback $a(y)=\frac{1}{T} G y$ exponentially stabilizes the origin of the "extended system"

$$
\left\{\begin{array}{l}
\dot{x}=\sum_{j=1}^{n} \tilde{f}_{j}(x) a_{j} \\
\dot{y}=\left(\sum_{k \in \mathbb{N}} \delta_{k T}\right)\left(x-y_{-\alpha}\right) 0<\alpha<T
\end{array}\right.
$$

the linear approximation of which, at the origin, is controllable. The matrix $G$ also determines the rate of exponential convergence of the solutions to the origin. From there, the remaining part of the construction may be seen as a technique for calculating an "equivalent" control for the system $\left(\bar{S}_{0}\right)$ which preserves this rate of convergence.

- Step 3. The partition $\left(P_{k}\right)_{(k=1, \ldots, K)}$ may not be unique since there may exist various ways of expressing a given vector field $\tilde{f}_{j}$ as a Lie bracket of the control vector fields $f_{i}$. Once again, a choice which may also affect the domain of robustness of the control has to be made.

- Step 4. Note that $\underline{C}(k) \leq \bar{C}(k)$. This inequality characterizes the main advantage of the c.l.a. framework over the f.l.a. one in terms of complexity. Indeed the "complexity" of the final control expression (25), as measured by the number of terms and time-periodic functions involved in it, increases with the numbers $C(k)$ equal to either $\underline{C}(k)$ or $\bar{C}(k)$, depending on the framework which is adopted. When $\underline{C}(k)$ is much smaller than $\bar{C}(k)$, this makes an important difference. However, contrary to the case of $\underline{C}(k)$, the value of $\bar{C}(k)$ is insensitive to "small" variations of the control vector fields $f_{i}$ associated with the control system $\left(S_{0}\right)$. It depends only on the value of $l(k)$, and on the set of indeterminates $\left\{X_{\tau_{1}^{k}}, \ldots, X_{\tau_{l(k)}^{k}}\right\}$ which are associated with every bracket in $P_{k}$. The same remark holds for the coefficients $\mu_{j}^{k, c}$ involved in the decomposition (23) of $\tilde{f}_{j}$ on a basis of $P_{k}$. It is precisely this property of independence associated with the f.l.a. framework which allows for the strong robustness property ii) of Theorem 2. For small values of $l(k)$, the determination of $\bar{C}(k)$ is simple. For example, $l(k)=2 \Rightarrow \bar{C}(k)=1$ (no possible repetition in this case), $l(k)=3 \Rightarrow \bar{C}(k)=2$ if the indeterminates $X_{\tau_{1}^{k}}, X_{\tau_{2}^{k}}$, and $X_{\tau_{3}^{k}}$ are all different, and $\bar{C}(k)=1$ if two of them are the same. The calculation of $\bar{C}(k)$ becomes rapidly more complicated when $l(k)$ increases.

From here on, the last steps of the construction will be commented upon in the c.l.a. case only. However, all properties that we shall discuss also hold in the f.l.a. case. The transposition of one case to the other just requires to interchange $f_{i}$ with the indeterminate $X_{i}$, and $\tilde{f}_{j}$ with $\mathcal{B}_{j}$. 
- Step 5. This is the central step of the construction. Let us first recall the definitions of a Minimally Cancelling (MC) set and of the property of independence with respect to an integer $p$ for a family of finite subsets of $\mathbb{R}[11,24]$.

Definition 1. $[11,24]$ Let $\Omega$ be a finite subset of $\mathbb{R}$ and $|\Omega|$ denote the number of elements of $\Omega$. The set $\Omega$ is said to be "Minimally Canceling" (in short, MC) if and only if:

i) $\sum_{\omega \in \Omega} \omega=0$

ii) this is the only zero sum with at most $|\Omega|$ terms taken in $\Omega$ with possible repetitions:

$$
\left.\begin{array}{c}
\sum_{\omega \in \Omega} \lambda_{\omega} \omega=0 \\
\sum_{\omega \in \Omega}\left|\lambda_{\omega}\right| \leq|\Omega| \\
\left(\lambda_{\omega}\right)_{\omega \in \Omega} \in \mathbb{Z}^{|\Omega|}
\end{array}\right\} \Longrightarrow\left\{\begin{array}{lll}
\left(\lambda_{\omega}\right)_{\omega \in \Omega} & =(0, \ldots, 0) \\
& \text { or }(1, \ldots, 1) \\
& \text { or }(-1, \ldots,-1) .
\end{array}\right.
$$

Definition 2. $[11,24]$ Let $\left(\Omega^{\xi}\right)_{\xi \in E}$ be a finite family of finite subsets $\Omega_{\xi}$ of $\mathbb{R}$. The family $\left(\Omega^{\xi}\right)_{\xi \in E}$ is said to be "independent with respect to $\mathrm{p}$ " if and only if:

$$
\left.\begin{array}{l}
\sum_{\xi \in E} \sum_{\omega \in \Omega^{\xi}} \lambda_{\omega} \omega=0 \\
\sum_{\xi \in E} \sum_{\omega \in \Omega^{\xi}}\left|\lambda_{\omega}\right| \leq p \\
\left(\lambda_{\omega}\right)_{\omega \in \Omega^{\xi}, \xi \in E} \in \mathbb{Z}^{\Sigma\left|\Omega^{\xi}\right|}
\end{array}\right\} \Longrightarrow \sum_{\omega \in \Omega^{\xi}} \lambda_{\omega} \omega=0 \quad \forall \xi \in E .
$$

The interest of MC sets in our context comes mainly from the following result proved by Kurzweil and Jarnik [9] (see also [11]).

Proposition 2. [9] Let $f_{1}, \ldots f_{l}$ be smooth vector fields, and $\alpha_{i}(i=1, \ldots, l)$ be integrable functions such that

$$
|I|<l \Longrightarrow \int_{0}^{T} \alpha_{I}=0
$$

Then,

$$
\begin{aligned}
& \sum_{\substack{\sigma(1), \ldots, \sigma(l)) \\
\sigma \in \mathfrak{S}(l)}} f_{I} \int_{0}^{T} \alpha_{I} \\
& =\frac{1}{l} \sum_{\substack{\sigma(1), \ldots, \sigma(l)) \\
\sigma \in \mathfrak{S}(l)}}\left[f_{\sigma(1)},\left[f_{\sigma(2)},\left[\ldots, f_{\sigma(l)}\right] \ldots\right]\right] \int_{0}^{T} \alpha_{I},
\end{aligned}
$$

with $\mathfrak{S}(l)$ denoting the group of permutation of l elements.

The stabilizing properties of the feedback control (25) much rely on the combination of the above proposition with a property of sine and cosine functions which is stated in the next proposition (the proof of which involves standard computations and is thus omitted). 
Proposition 3. Let $\Omega=\left\{\omega_{1}, \ldots, \omega_{l}\right\}$ denote a $M C$ set such that the $\omega_{j}$ 's have a common divisor $\bar{\omega}$ $\left(=\frac{2 \pi}{T}\right)$. Define also

$$
\alpha_{i}(t)= \begin{cases}\cos \omega_{i} t & (i=1) \\ \sin \omega_{i} t & (i=2, \ldots, l)\end{cases}
$$

Then, for any $I=(\sigma(1), \ldots, \sigma(p))(\sigma \in \mathfrak{S}(p))$, with $|I|=p \leq l$

$$
\int_{0}^{T} \alpha_{I}=\left\{\begin{array}{cl}
0 & \text { if }|I|<l \\
\frac{(-1)^{l-1} T}{2^{l-1}} \frac{1}{\omega_{\sigma(1)}\left(\omega_{\sigma(1)}+\omega_{\sigma(2)}\right) \cdots\left(\omega_{\sigma(1)}+\ldots+\omega_{\sigma(l-1)}\right)} & \text { if }|I|=l .
\end{array}\right.
$$

The important relation which results from the combination of the above two propositions is the following

$$
\begin{gathered}
\sum_{\substack{\sigma(1), \ldots, \sigma(l)) \\
\sigma \in \mathfrak{S}(l)}} f_{I} \int_{0}^{T} \alpha_{I} \\
=\frac{(-1)^{l-1} T}{l 2^{l-1}} \sum_{\substack{\sigma(1), \ldots, \sigma(l)) \\
\sigma \in \mathfrak{S}(l)}} \frac{\left[f_{\sigma(1)},\left[f_{\sigma(2)},\left[\ldots, f_{\sigma(l)}\right] \ldots\right]\right]}{\omega_{\sigma(1)}\left(\omega_{\sigma(1)}+\omega_{\sigma(2)}\right) \cdots\left(\omega_{\sigma(1)}+\ldots+\omega_{\sigma(l-1)}\right)} \\
I=
\end{gathered}
$$

The existence of MC sets $\Omega^{k, c}$ which fulfill conditions i) and iii) of Step 5 is proved in [11]. Furthermore, it is shown in this reference that the set of admissible sets $\Omega^{k, c}$ (seen as a subset of $\mathbb{R}^{\sum_{k} l(k) C(k)}$ ) is open. Therefore, each element of $\Omega^{k, c}$ can be chosen rational, and the existence of a common divisor is in this case automatically ensured by the fact that any finite set of rational numbers has a common divisor.

We may remark that condition iii) is generically satisfied. This is partly due to the fact that, within the set of finite-dimensional matrices of real numbers the subset of full-ranked matrices of rational numbers is dense (more details about this issue can be found in [11]). As a consequence, a typical way of carrying out Step 5 consists in first determining one family of MC sets composed of rational numbers, such that this family is independent w.r.t. $\max _{k \in\{1, \ldots, K\}} l(k)$, and then verifying for each $k \in\{1, \ldots, K\}$ that the third condition is satisfied. Also, once the common divisor $\bar{\omega}_{1}$ associated with this family has been determined, one can subsequently impose another value $\bar{\omega}_{2}$ by multiplying all elements in the sets $\Omega^{k, c}$ by $\frac{\bar{\omega}_{2}}{\bar{\omega}_{1}}$. A further possible requirement, which tends to complicate the procedure, is to have the smallest element in the sets $\Omega^{k, c}$ equal to the common divisor of the family.

- Step 6. Let $j \in\{1, \ldots, n\}$. If $\mathcal{B}_{j} \in P_{k}$, then $\tilde{f}_{j}$ belongs to the $C(k)$-dimensional vector space generated by the vector fields

$$
\left[f_{\tau_{\sigma(1)}^{k}},\left[f_{\tau_{\sigma(2)}^{k}},\left[\ldots, f_{\tau_{\sigma(l(k))}^{k}}\right] \ldots\right]\right] \quad(\sigma \in \mathfrak{S}(l(k)))
$$

Since the vector fields $g^{k, c}(c \in\{1, \ldots, C(k)\})$ defined in (21) also belong, by construction, to this space, and since they are linearly independent (by Step 5 ), they form a basis and $\tilde{f}_{j}$ can be expressed as a linear combination of them. Relation (23) just indicates that the coefficients $\mu_{j}^{k, c}$, multiplied by a given factor, are the coefficients of this decomposition. Note that, in the context of the present construction, another advantage of working in the f.l.a. framework comes from that this set of coefficients can be determined beforehand, since it is independent of the vector fields $f_{i}$ themselves. 
At this point of the construction, one deduces from $(21,23,30)$, that

$$
\mathcal{B}_{j} \in P_{k} \Rightarrow \tilde{f}_{j}=\frac{1}{T} \sum_{c=1}^{C(k)} \mu_{j}^{k, c} \sum_{\substack{\left(\tau_{\sigma(1)}^{k}, \ldots, \tau_{\sigma(l(k))}^{k}\right) \\ \sigma \in \mathfrak{S}(l(k))}} f_{I} \int_{0}^{T} \alpha_{I}^{k, c}
$$

- Step 7. There are many possible choices for the functions $v$. For example, one can take

$$
v_{\tau_{i}^{k}}^{k, c}(x)=\left\{\begin{array}{cl}
\left|\sum_{j: \mathcal{B}_{j} \in P_{k}} \mu_{j}^{k, c} a_{j}(x)\right|^{\frac{1}{l(k)}} \operatorname{sign}\left(\sum_{j: \mathcal{B}_{j} \in\left\{P_{k}\right\}} \mu_{j}^{k, c} a_{j}(x)\right) & (i=1) \\
\left.\sum_{j: \mathcal{B}_{j} \in P_{k}} \mu_{j}^{k, c} a_{j}(x)\right|^{\frac{1}{(k)}} & (i=2, \ldots, l(k)) .
\end{array}\right.
$$

Finally, note that, in view of $(24,30,31)$,

$$
\begin{aligned}
& \sum_{k=1}^{K} \sum_{c=1}^{C(k)} \sum_{I=\left(\tau_{\sigma(1)}^{k}, \ldots, \tau_{\sigma(l(k))}^{k}\right)} f_{I} i d(x) \int_{0}^{T}(\alpha v)_{I}^{k, c}(x)=T \sum_{k=1}^{K} \sum_{j: \mathcal{B}_{j} \in P_{k}} \tilde{f}_{j}(x) a_{j}(x) \\
& \sigma \in \mathfrak{S}(l(k)) \\
& =T \sum_{j=1}^{n} \tilde{f}_{j}(x) a_{j}(x) .
\end{aligned}
$$

\subsection{Control design from a homogeneous approximation}

It is often convenient and simpler to work with approximations of control systems. For instance, linear approximations are commonly used for feedback control design when they are controllable (or at least stabilizable). When the linear approximation of the system, evaluated at the equilibrium which feedback control is in charge of stabilizing, is not stabilizable, the extension of the notion of linear approximation yields to homogeneous controllable approximations. Using such an approximation is particularly well adapted to the design of continuous homogeneous feedbacks which render the closed-loop system homogeneous of degree zero. The reason is that asymptotic stabilization of the origin of the homogeneous approximation automatically ensures that the origin of the initial control system is also asymptotically (locally) stabilized by the same feedback control law. It is however important to realize that this property does not necessarily hold when using hybrid controllers such as those which we are considering here. It is not difficult to work out simple examples which illustrate this fact. However, we show in this section that a robust controller for the system $\left(S_{0}\right)$ can be derived from the knowledge of a homogeneous approximation of this system, provided that some extra condition is satisfied by the control law. This condition is stated in a theorem, after recalling a few definitions and properties about homogeneous systems. A complementary proposition indicates how the control design algorithm previously described can be completed in order to cope with the use of homogeneous approximations.

Given $\lambda>0$ and a weight vector $r=\left(r_{1}, \ldots, r_{n}\right)\left(r_{i}>0 \forall i\right)$, a dilation $\delta_{\lambda}^{r}$ is a map from $\mathbb{R}^{n}$ to $\mathbb{R}^{n}$ defined by:

$$
\delta_{\lambda}^{r}\left(z_{1}, \ldots, z_{n}\right)=\left(\lambda^{r_{1}} z_{1}, \ldots, \lambda^{r_{n}} z_{n}\right) .
$$


A function $f \in \mathcal{C}^{0}\left(\mathbb{R}^{n} ; \mathbb{R}\right)$ is homogeneous of degree $l$ with respect to the family of dilations $\delta_{\lambda}^{r}(\lambda>0)$, or, more concisely, $\delta^{r}$-homogeneous of degree $l$, if

$$
\forall \lambda>0, \quad f\left(\delta_{\lambda}^{r}(z)\right)=\lambda^{l} f(z)
$$

A $\delta^{r}$-homogeneous norm can be defined as a positive definite function of $x, \delta^{r}$-homogeneous of degree one. Although this is not a "true" norm when the weight coefficients are not all equal, it still provides a means of "measuring" the size of $x$.

A continuous vector field $X$ on $\mathbb{R}^{n}$ is $\delta^{r}$-homogeneous of degree $d$ if, for all $i=1, \ldots, n$, the function $x \longmapsto X_{i}(x)$ is $\delta^{r}$-homogeneous of degree $r_{i}+d$. According to these definitions, homogeneity is coordinate dependent, however it is possible to define the above concepts in a coordinate independent framework [7,19].

The following property is used extensively in the sequel. Given a family of dilations $\delta_{\lambda}^{r}(\lambda>0)$, a smooth function $f$ and a smooth vector field $X, \delta^{r}$-homogeneous of degree $\operatorname{deg}(f)$ and $\operatorname{deg}(X)$ respectively, the function $X f$ is $\delta^{r}$-homogeneous of degree

$$
\operatorname{deg}(X f)=\operatorname{deg}(X)+\operatorname{deg}(f)
$$

Finally, we say that the system

$$
\dot{z}=\sum_{i=1}^{m} b_{i}(z) u_{i}
$$

is a $\delta^{r}$-homogeneous approximation of $\left(S_{0}\right)$ if:

1. the change of coordinates $\phi: x \longmapsto z$ transforms $\left(S_{0}\right)$ into

$$
\dot{z}=\sum_{i=1}^{m}\left(b_{i}(z)+g_{i}(z)\right) u_{i}
$$

where $b_{i}$ is $\delta^{r}$-homogeneous of some degree $d_{i}<0$, and $g_{i}$ denotes higher-order terms, i.e. such that $g_{i, j}$ (the $j$-th component of $g_{i}$ ) satisfies

$$
g_{i, j}=o\left(\rho^{r_{j}+d_{i}}\right), \quad(j=1, \ldots, n)
$$

where $\rho$ is a $\delta^{r}$-homogeneous norm;

2. the system (34) is controllable.

Hermes [5], and Stefani [22] have shown that any driftless system $\left(S_{0}\right)$ satisfying the LARC (Lie Algebra Rank Condition) at the origin (2) has a homogeneous approximation. Homogeneous approximations of controllable driftless systems are not unique in general. Explicit construction of such approximations requires to find both a weight vector and a change of coordinates for which properties 1 and 2 above are fulfilled. A constructive procedure can be found in [22,23] (see also [5] but with a less explicit change of coordinates).

A particular homogeneous approximation of $\left(S_{0}\right)$ corresponds to the case when the vector fields $b_{i}$ are all homogeneous of the same degree -1 . Let us briefly recall some features of this approximation. 
Consider the control filtration $\mathcal{F}$ of $\operatorname{Lie}\left(f_{1}, \ldots, f_{m}\right)$, defined as $\mathcal{F} \triangleq\left(\mathcal{F}_{j}\right)_{j \geq 0}$ with

$$
\begin{aligned}
& \mathcal{F}_{0} \triangleq\{0\} \\
& \mathcal{F}_{1} \triangleq \operatorname{span}\left\{f_{1}, \ldots, f_{m}\right\} \\
& \mathcal{F}_{2} \triangleq \operatorname{span}\left\{f_{1}, \ldots, f_{m},\left[f_{1}, f_{2}\right], \ldots,\left[f_{1}, f_{m}\right], \ldots,\left[f_{m-1}, f_{m}\right]\right\} \\
& \vdots \\
& \mathcal{F}_{k} \triangleq \operatorname{span}\left\{\text { all Lie brackets of the } f_{i}^{\prime} s \text { of length } \ell \leq k\right\} \\
& \text {. }
\end{aligned}
$$

Denote also

$$
F_{k}(0) \triangleq \operatorname{span}\left\{f(0): f \in \mathcal{F}_{k}\right\}
$$

and $n_{k} \triangleq \operatorname{dim} F_{k}(0)$. Then, by the LARC at the origin, there exists a smallest integer $P$ such that

$$
0=n_{0}<n_{1} \leq n_{2} \leq \ldots \leq n_{P-1}<n_{P}=n .
$$

Now, define the weight vector $r$ according to

$$
r_{j} \triangleq p \quad \text { for } n_{p-1}+1 \leq j \leq n_{p} \quad(p=1, \ldots, P) .
$$

Note that the sequence $r_{1}, r_{2}, \ldots, r_{n}$ is increasing, i.e. $1=r_{1} \leq r_{2} \leq \ldots \leq r_{n}=P$. The results given in $[5,22]$ imply:

Proposition 4. [5, 22] There exists a $\delta^{r}$-homogeneous approximation (34) of system $\left(S_{0}\right)$ with $r$ given by (37). Furthermore, every control vector field of the approximating system is $\delta^{r}$-homogeneous of degree -1 .

We refer the reader to the references cited above for the construction of a change of coordinates $\phi$ that transforms $\left(S_{0}\right)$ into $(35)$. Let us remark that the control vector fields $b_{i}$ of the approximating homogeneous system (34) are polynomials in the $z$ coordinates, and that $\left\{b_{1}, \ldots, b_{m}\right\}$ forms a nilpotent set of vector fields - more precisely, any Lie bracket of the $b_{i}$ 's of length strictly larger than $P$ is identically zero.

Since homogeneous approximations are nilpotent, for any time-function $u$, the Chen-Fliess series associated with any such an approximation only involves a finite number of terms. This property is useful when trying to derive exponential stabilizers for the homogeneous approximation of a given system. Of course, such controllers are of interest only if they are also exponential stabilizers for the original system. The following theorem points out sufficient conditions on the control law to ensure that such is the case.

Theorem 3. Consider a $\delta^{r}$-homogeneous approximation (34) of $\left(S_{0}\right)$, with $d_{i} \triangleq \operatorname{deg}\left(b_{i}\right)(i=1, \ldots, m)$, and a control function $u \in \mathcal{C}^{0}\left(U \times \mathbb{R}^{+} ; \mathbb{R}^{m}\right)$ such that the three assumptions in Theorem 1 are verified for this approximating system. Assume furthermore that the following assumption, which is a stronger version of the third assumption in Theorem 1, is also verified for the approximating system:

3-bis. for any multi-index $I=\left(i_{1}, \ldots, i_{|I|}\right)$ of length $|I| \leq 1 / \alpha$,

$$
\int_{0}^{T} u_{I}(z)=\sum_{k: r_{k} \geq\|I\|} a_{I, k} z_{k}+o(z)
$$

where $\|I\| \triangleq-\sum_{j=1}^{|I|} d_{i_{j}}$, and the $a_{I, k}$ 's are some scalars.

Then, the three assumptions of Theorem 1 are verified for the system (35). 
Note that it is not required that all $d_{i}$ 's be equal, as it occurs when the approximation is obtained by using the dilation defined by (37).

When applying the algorithm of Section 4.1 to the approximation (34), the control law $u$ given by (25) may not satisfy the extra condition 3-bis of Theorem 3. However, it is possible to impose extra requirements on the matrix $G$ defined in Step 2 so as to guarantee the satisfaction of this condition. For instance, the following result is proved in [17].

Proposition 5. Consider a $\delta^{r}$-homogeneous approximation (34) of $\left(S_{0}\right)$, with every control vector field of this system being $\delta^{r}$-homogeneous of degree -1 . Without loss of generality, we assume that the variables $z_{i}$ are ordered by increasing weight, i.e.

$$
r_{1} \leq r_{2} \leq \ldots \leq r_{n}
$$

and decompose $z$ as $z=\left(z^{1}, \ldots, z^{P}\right)$, where each $z^{p}(1 \leq p \leq P)$ is the sub-vector of $z$ whose components have same weight $r^{p}\left(r_{1} \leq r^{p} \leq r_{n}\right)$ with

$$
r_{1}=r^{1}<r^{2}<\ldots<r^{P}=r_{n} .
$$

Consider the control design algorithm described in Section 4.1 and applied to (34).

Let $\tilde{b}_{j}(j \in\{1, \ldots, n\})$ denote the vector fields defined according to Step 1 of the algorithm, and

$$
\tilde{B}(z) \triangleq\left(\tilde{b}_{1}(z), \ldots, \tilde{b}_{n}(z)\right) .
$$

Due to the ordering of the variables $z_{i}$, the matrix $\tilde{B}(z)$ is block lower triangular, and block diagonal at $z=0$, i.e.

$$
\tilde{B}(0)=\left(\begin{array}{cccc}
\tilde{B}^{11} & 0 & \cdots & 0 \\
0 & \tilde{B}^{22} & & \vdots \\
\vdots & \vdots & \ddots & 0 \\
0 & \cdots & \cdots & \tilde{B}^{P P}
\end{array}\right) .
$$

Assume that the control gain matrix $G$ involved in Step 2 of the algorithm is chosen as follows

$$
G=\tilde{B}(0)^{-1}\left(H-I_{n}\right)
$$

with the matrix $H$ being block upper triangular, i.e.

$$
A=\left(\begin{array}{cccc}
H^{11} & \star & \cdots & \star \\
0 & H^{22} & \ddots & \vdots \\
\vdots & \ddots & \ddots & \star \\
0 & \cdots & 0 & H^{P P}
\end{array}\right)
$$

and discrete-stable $\left(\Leftrightarrow H^{i i}\right.$ is discrete-stable for $\left.i \in\{1, \ldots, P\}\right)$.

Then, the three assumptions of Theorem 1 are verified for the system $\left(S_{0}\right)$.

\subsection{Robust exponential stabilizers for chained systems}

In some cases, it is possible to take advantage of specific structural properties associated with the control system under consideration, in order to derive robust control laws that are simpler than those obtained by application of the general algorithm presented in Section 4.1. 
We illustrate this possibility in the case of the following $n$-dimensional chained system

$$
\left\{\begin{aligned}
\dot{x}_{1} & =u_{1} \\
\dot{x}_{2} & =u_{2} \\
\dot{x}_{3} & =u_{1} x_{2} \\
& \vdots \\
\dot{x}_{n} & =u_{1} x_{n-1}
\end{aligned}\right.
$$

The next result points out a set of robust exponential stabilizers for this system.

Theorem 4. With the control function $u \in \mathcal{C}^{0}\left(\mathbb{R}^{n} \times \mathbb{R}^{+} ; \mathbb{R}^{2}\right)$ defined by

$$
\left\{\begin{array}{l}
u_{1}(x, t)=\frac{1}{T}\left[\left(k_{1}-1\right) x_{1}+2 \pi \rho_{q}(x) \sin (\omega t)\right] \\
u_{2}(x, t)=\frac{1}{T}\left[\left(k_{2}-1\right) x_{2}+\sum_{i=3}^{n} 2^{i-2}(i-2) !\left(k_{i}-1\right) \frac{x_{i}}{\rho_{q}^{i-2}(x)} \cos ((i-2) \omega t)\right]
\end{array}\right.
$$

with

$$
\begin{aligned}
& T=2 \pi / \omega \quad(\omega \neq 0), \\
& \rho_{q}(x)=\sum_{j=3}^{n} a_{j}\left|x_{j}\right|^{\frac{1}{q+j-2}}, \quad\left(q \geq n-2, a_{j}>0\right), \\
& \left|k_{i}\right|<1, \quad \forall i=1, \ldots, n,
\end{aligned}
$$

the three assumptions in Theorem 1, and the extra assumption in Theorem 3, are verified for the system (39).

Corollary 1. (of Ths. 3 and 4) With the control function (40), the three assumptions in Theorem 1 are verified for any analytic driftless system for which the chained system (39) is a $\delta^{r}$-homogeneous approximation, with $r=(1, q, \ldots, q+n-2)$ and $q \geq n-2$.

\section{FinAL REMARKS}

We conclude the present study with a few general remarks. The first one concerns the assumption of analycity which has been made on the control vector fields of the system $\left(S_{0}\right)$. In fact, the main results of the study remain valid when the control vector fields are smooth only, or even of class $\mathcal{C}^{k}$ with $k$ large enough depending on the structure of the system's Control Lie Algebra. The proofs can be carried out in the same manner except for mild complications which arise in particular from using a finite expansion of the control system's solutions instead of the infinite Chen-Fliess expansion. Such a finite expansion can be derived in the same way as a Taylor expansion with integral remainder is obtained for a smooth function.

The second remark concerns possible applications of Theorem 1 in order to construct robust exponential stabilizers. In Section 4, this result was combined with the use of sinusoidal functions of time in the control expression. However, there is no obligation for the control law to depend on time in this manner. For instance, when the system $\left(S_{0}\right)$ is known to be differentially flat [4], adequate control functions can be obtained by considering specifically tailored flatness-based solutions to the open-loop steering problem, as done for example in [1] in the case of chained systems. Is is worth mentioning at this point that, although the control design approach and robustness analysis in [1] are very different from the ones developed in the present paper, the specific conditions imposed in this reference on the control law imply that the assumptions of Theorem 1 are verified. This suggests that these assumptions are not unduly strong and also illustrates the fact that the domain of application of Theorem 1 extends to different control design techniques.

How does the general control design procedure described in Section 4.1 compare with the related one developed in [15] for the design of exponentially stabilizing continuous time-periodic feedbacks? Besides the 
fact, already pointed out before, that the latter method fails to produce controls which are robust in the sense considered in the present paper, the number of calculations required to synthesize the control law is also generally much higher and the resulting control expression significantly more complicated (because of state dependent terms which only vanish when the state is constant). Periodic dependence with respect to time also involves high frequencies resulting in highly oscillatory trajectories (a feature rarely desirable when dealing with mechanical systems), whereas the construction here proposed allows for choosing the control frequencies independently of the asymptotic rate of convergence.

Nonetheless, we are also aware that the hybrid open-loop/feedback controls here considered carry their own limitations the importance of which remains to be evaluated in future studies. For instance, just to cite a slightly uncommon issue, robustness with respect to modeling errors has been obtained under the assumption that the updating period of the control, i.e. the time interval during which the control is applied in open-loop fashion, is an exact multiple of the periods of the time functions involved in the control law. It is possible to show (this is beyond the scope of this study) that the slightest violation of this assumption, while unavoidable in practice for reasons that anyone having control implementation in mind will easily figure out, almost invariably results in the loss of stability of the origin of the controlled system. This means that the control is not robust with respect to the imperfect verification of this assumption. Although the source of this robustness problem is little connected with the modeling of the control system itself, its practical consequences should probably not be disregarded when attempting to address the complex and delicate issue of comparing different control techniques.

\section{APPENDIX}

\section{Proof of Theorem 1}

The proof much relies on the following lemma.

Lemma 1. Given $\epsilon_{1}>0$, there exists $\delta>0$ such that, for $\left|x_{0}\right| \leq \delta$ and $|\epsilon| \leq \epsilon_{1}$, the solution of

$$
\dot{x}=\sum_{i=1}^{m}\left(f_{i}(x)+h_{i}(\epsilon, x)\right) u_{i}\left(x_{0}, t\right), \quad x(0)=x_{0}
$$

is defined on $[0, T]$ and satisfies

$$
x(T)=A x_{0}+\beta\left(\epsilon, x_{0}\right)+\gamma\left(\epsilon, x_{0}\right)+o\left(x_{0}\right)
$$

with

$$
\begin{aligned}
& \frac{\left|\beta\left(\epsilon, x_{0}\right)\right|}{\left|x_{0}\right|} \rightarrow 0 \text { as } \epsilon \rightarrow 0 \text { uniformly in } x_{0}\left(\left|x_{0}\right| \leq \delta\right) \\
& \frac{\left|\gamma\left(\epsilon, x_{0}\right)\right|}{\left|x_{0}\right|} \rightarrow 0 \text { as } x_{0} \rightarrow 0 \text { uniformly in } \epsilon\left(|\epsilon| \leq \epsilon_{1}\right) .
\end{aligned}
$$

The proof of this lemma is given at the end of this section.

Existence of the solutions of the system $\left(\bar{S}_{\epsilon}\right)$ controlled by $u(y, t)$, when the initial conditions $x_{0}$ and $y_{0}$ are close enough to the origin, can easily be established (details are left to the interested reader) once it is proven that these solutions at least exist on a small time-interval and are uniformly bounded with respect to $x_{0}$ and $y_{0}$. Existence on a small time-interval is in fact granted by application of Proposition 1 (as illustrated in the proof of Lem. 1), while uniform boundedness of the solutions simply results from the property of (local) stability which is proven below. 
In order to prove that the origin of the system $\left(\bar{S}_{\epsilon}\right)$ controlled by $u(y, t)$ is locally (uniformly) exponentially stable, one must establish that i) the solutions of this system converge exponentially to zero (uniformly with respect to the initial conditions $\left.\left(t_{0}, x_{0}, y_{0}\right)\right)$, and ii) the origin of the controlled system is stable.

Since $A$ is a discrete-stable matrix, there exists a positive definite matrix $P$ such that $|A|_{P}<1 \Leftrightarrow \exists \tau<1$ : $\left.|A x|_{P} \leq \tau|x|_{P}\right)$. For the sake of simplifying the notation, and keeping in mind that subsequent normed vectors will refer to the norm induced by the matrix $P$, we will just drop the index $P$ in the remaining of the proof.

Exponential convergence: Let $\left(x_{\epsilon}\left(., t_{0}, x_{0}, y_{0}\right), y_{\epsilon}\left(., t_{0}, x_{0}, y_{0}\right)\right)$ denote the solution of the controlled system $\left(\bar{S}_{\epsilon}\right)$ with initial conditions $\left(t_{0}, x_{0}, y_{0}\right), t_{0} \in\left[k_{0} T,\left(k_{0}+1\right) T\right), k_{0} \in \mathbb{N}$. Then for any $k \in \mathbb{N}$ such that $t_{0} \leq k T$, and any $t \in[k T,(k+1) T)$, this solution satisfies

$$
\left\{\begin{array}{l}
\dot{x}=\sum_{i=1}^{m}\left(f_{i}(x)+h_{i}(\epsilon, x)\right) u_{i}(x(k T), t) \\
\dot{y}=0, \quad y(t)=x(k T) .
\end{array}\right.
$$

In order to show the exponential convergence to zero of the trajectories of (46), it is clearly sufficient to show that the $x$ component converges exponentially to the origin. In view of relations (43-45) in Claim 1, there exist $\epsilon_{0}>0, \delta_{0}>0$, and a positive real number $\tau<1$ such that, for $\left|x_{0}\right| \leq \delta_{0}$ and $\epsilon \leq \epsilon_{0}$

$$
\left|x_{\epsilon}\left((k+1) T, t_{0}, x_{0}, y_{0}\right)\right| \leq \tau\left|x_{\epsilon}\left(k T, t_{0}, x_{0}, y_{0}\right)\right|, \quad \forall k>k_{0} .
$$

This already establishes that the sequence $\left\{x_{\epsilon}\left(k T, t_{0}, x_{0}, y_{0}\right)\right\}_{k \in \mathbb{N}}$ converges exponentially to zero, uniformly with respect to the initial conditions $\left(t_{0}, x_{0}, y_{0}\right)$.

In order to infer uniform exponential convergence to zero of $x_{\epsilon}\left(t, t_{0}, x_{0}, y_{0}\right)$, it is thus sufficient to show that

$$
\left|x_{\epsilon}\left(k T+s, t_{0}, x_{0}, y_{0}\right)\right| \leq K\left|x_{\epsilon}\left(k T, t_{0}, x_{0}, y_{0}\right)\right|^{\eta}, \quad \forall s \in[0, T), \forall k>k_{0},
$$

for some positive constants $K$ and $\eta$ independent of $t_{0}, x_{0}, y_{0}$. Since $u$ is $T$-periodic

$$
\begin{aligned}
x_{\epsilon}\left(k T+s, t_{0}, x_{0}, y_{0}\right) & =x_{\epsilon}\left(s, 0, x_{\epsilon}\left(k T, t_{0}, x_{0}, y_{0}\right), y_{\epsilon}\left(k T, t_{0}, x_{0}, y_{0}\right)\right) \\
& =x_{\epsilon}\left(s, 0, x_{\epsilon}\left(k T, t_{0}, x_{0}, y_{0}\right), x_{\epsilon}\left(k T, t_{0}, x_{0}, y_{0}\right)\right) .
\end{aligned}
$$

Therefore (48) is equivalent to

$$
\left|x_{\epsilon}\left(s, 0, x_{0}, x_{0}\right)\right| \leq K\left|x_{0}\right|^{\eta}, \quad \forall s \in[0, T) .
$$

From Assumption 1, and the continuity of the vector fields $f_{i}$, and $h_{i}$, the above inequality follows by the classical Gronwall lemma.

Uniform stability of the origin: We distinguish two cases, according to whether $t_{0}$ is, or is not, a multiple of $T$.

Case 1: $t_{0}$ is not a multiple of $T$.

Then there exists $k_{0} \in \mathbb{N}$ such that $k_{0} T<t_{0}<\left(k_{0}+1\right) T$. There also exists an open ball $B_{\epsilon} \in \mathbb{R}^{n}$ centered on 0 such that the function $\left(t, t_{0}, x_{0}, y_{0}\right) \mapsto x_{\epsilon}\left(t, t_{0}, x_{0}, y_{0}\right)$ is continuous on the set $\left\{\left(t, t_{0}, x, y\right): t_{0} \leq t \leq\right.$ $\left.\left(k_{0}+1\right) T, t_{0} \in\left(k_{0} T,\left(k_{0}+1\right) T\right), x \in B_{\epsilon}, y \in B_{\epsilon}\right\}$. Therefore, the function $\nu_{\epsilon}$ defined by

$$
\nu_{\epsilon}\left(x_{0}, y_{0}\right) \triangleq \sup _{t_{0} \in\left(k_{0} T,\left(k_{0}+1\right) T\right)} \sup _{t \in\left[t_{0},\left(k_{0}+1\right) T\right)}\left|x_{\epsilon}\left(t, t_{0}, x_{0}, y_{0}\right)\right|
$$

is itself continuous on $B_{\epsilon} \times B_{\epsilon}$. Note that $\nu_{\epsilon}(0,0)=0$, since $x_{\epsilon}\left(t, t_{0}, 0,0\right)=0, \forall t \geq t_{0}$, and that $\nu\left(x_{0}, y_{0}\right) \geq\left|x_{0}\right|$. Furthermore, there exists $\delta_{\epsilon}>0$ such that $\nu_{\epsilon}\left(x_{0}, y_{0}\right)<\min \left(1, r_{B_{\epsilon}}\right), \forall\left(x_{0}, y_{0}\right):\left|x_{0}\right| \leq \delta_{\epsilon},\left|y_{0}\right| \leq \delta_{\epsilon}$, with $r_{B_{\epsilon}}$ 
denoting the radius of $B_{\epsilon}$. Recall also that

$$
\left|y_{\epsilon}\left(t, t_{0}, x_{0}, y_{0}\right)\right|=\left|y_{0}\right|, \forall t_{0} \in\left(k_{0} T,\left(k_{0}+1\right) T\right), \forall t \in\left[t_{0},\left(k_{0}+1\right) T\right) .
$$

Now, since by (47) and (48)

$$
\left|x_{\epsilon}\left(t, t_{0}, x_{0}, y_{0}\right)\right| \leq K\left|x_{\epsilon}\left(\left(k_{0}+1\right) T, t_{0}, x_{0}, y_{0}\right)\right|^{\eta}, \quad \forall t \geq\left(k_{0}+1\right) T,
$$

and

$$
\left|y_{\epsilon}\left(t, t_{0}, x_{0}, y_{0}\right)\right| \leq\left|x_{\epsilon}\left(\left(k_{0}+1\right) T, t_{0}, x_{0}, y_{0}\right)\right|, \quad \forall t \geq\left(k_{0}+1\right) T,
$$

one deduces from (50) and (51) (using also the fact that $|(x, y)| \leq|x|+|y|)$ that

$$
\begin{aligned}
& \left|x_{\epsilon}\left(t, t_{0}, x_{0}, y_{0}\right), y_{\epsilon}\left(t, t_{0}, x_{0}, y_{0}\right)\right| \leq K \nu_{\epsilon}\left(x_{0}, y_{0}\right)^{\eta}+\max \left(\nu_{\epsilon}\left(x_{0}, y_{0}\right),\left|y_{0}\right|\right), \\
& \forall t \geq t_{0}, \forall\left(x_{0}, y_{0}\right):\left|x_{0}\right| \leq \delta_{\epsilon},\left|y_{0}\right| \leq \delta_{\epsilon} .
\end{aligned}
$$

Case 2: $t_{0}$ is a multiple of $T$ (i.e. $\left.t_{0}=k_{0} T\right)$.

Then $y(0)=x_{0}$, and one easily obtains in this case

$$
\left|x_{\epsilon}\left(t, t_{0}, x_{0}, y_{0}\right), y_{\epsilon}\left(t, t_{0}, x_{0}, y_{0}\right)\right| \leq K\left|x_{0}\right|^{\eta}+\left|x_{0}\right|, \quad \forall t \geq t_{0}, \forall\left(x_{0}, y_{0}\right):\left|x_{0}\right| \leq \delta_{\epsilon},\left|y_{0}\right| \leq \delta_{\epsilon} .
$$

The comparison of the right-hand sides of inequalities (52) and (53) shows that (52) holds in fact for every value of $t_{0}$. To prove Theorem 1 , there only remains to prove Lemma 1.

Proof of Lemma 1. Let us first consider the issue of existence of the solutions of system (42). This system can equivalently be written as a system in $\mathbb{R}^{n+1}$ with $(x, \epsilon)$ as state vector, and $\left(u, u_{m+1} \equiv 0\right)$ as control vector:

$$
\left\{\begin{array}{l}
\dot{x}=\sum_{i=1}^{m}\left(f_{i}(x)+h_{i}(\epsilon, x)\right) u_{i}\left(x_{0}, t\right) \\
\dot{\epsilon}=u_{m+1} \triangleq 0
\end{array}\right.
$$

By applying Proposition 1 to this system, one deduces that for any compact set $S \times\left[-\epsilon_{1}, \epsilon_{1}\right], S \subset U$, there exists $\mu>0$ such that, if (14) and (15) are satisfied, the solution of (42) is defined on $[0, T]$ and can be expanded in the form of a Chen-Fliess series. Using Assumption 1 of Theorem 1, which implies that $|u(x, t)|$ tends to zero as $|x|$ tends to zero, one also deduces that for some positive $\delta,(14)$ and (15) are satisfied if $\left|x_{0}\right| \leq \delta$. Existence (and uniqueness) of the solutions of (42) is therefore guaranteed, and these solutions can be expanded, on $[0, T]$, as

$$
x(t)=x_{0}+\sum_{I}\left(\left(f+h_{\epsilon}\right)_{I} i d\right)\left(x_{0}\right) \int_{0}^{t} u_{I}\left(x_{0}\right),
$$

with $h_{\epsilon, i}(.) \triangleq h_{i}(\epsilon,$.$) . We may rewrite this equality as$

$$
x(t)=x_{0}+\sum_{I}\left(f_{I} i d\right)\left(x_{0}\right) \int_{0}^{t} u_{I}\left(x_{0}\right)+\sum_{I}\left(d_{I}^{h_{\epsilon}} i d\right)\left(x_{0}\right) \int_{0}^{t} u_{I}\left(x_{0}\right) .
$$

Here, $d_{I}^{h_{\epsilon}}=d_{i_{1}} \cdots d_{i_{k}}$ (for $\left.I=\left(i_{1}, \ldots, i_{k}\right)\right)$, with $d_{i}$ taken in $\left\{f_{i}, h_{\epsilon, i}\right\}$ and the product $d_{I}^{h_{\epsilon}}$ involving at least one $h_{\epsilon, i}$. Note that each series in (54) is convergent uniformly w.r.t. $x_{0}\left(\left|x_{0}\right| \leq \delta^{\prime}, \delta^{\prime}\right.$ possibly smaller that $\left.\delta\right), \epsilon$ 
$\left(|\epsilon| \leq \epsilon_{1}\right)$, and $t \in[0, T]$. This simply results from the existence (previously established) of $x(t)$, for $t \in[0, T]$, and the fact that the first series is convergent since

$$
x_{0}+\sum_{I}\left(f_{I} i d\right)\left(x_{0}\right) \int_{0}^{t} u_{I}\left(x_{0}\right)
$$

is precisely the Chen-Fliess expansion associated with $\left(S_{0}\right)$. Moreover, by Assumption 2 of Theorem 1,

$$
x_{0}+\sum_{I}\left(f_{I} i d\right)\left(x_{0}\right) \int_{0}^{T} u_{I}\left(x_{0}\right)=A x_{0}+o\left(x_{0}\right) .
$$

Let us now define $\beta\left(\epsilon, x_{0}\right)$ and $\gamma\left(\epsilon, x_{0}\right)$ as follows

$$
\beta\left(\epsilon, x_{0}\right) \triangleq \sum_{|I| \leq 1 / \alpha}\left(d_{I}^{h_{\epsilon}} i d\right)\left(x_{0}\right) \int_{0}^{T} u_{I}\left(x_{0}\right), \quad \gamma\left(\epsilon, x_{0}\right) \triangleq \sum_{|I|>1 / \alpha}\left(d_{I}^{h_{\epsilon}} i d\right)\left(x_{0}\right) \int_{0}^{T} u_{I}\left(x_{0}\right),
$$

so that

$$
\sum_{I}\left(d_{I}^{h_{\epsilon}} i d\right)\left(x_{0}\right) \int_{0}^{T} u_{I}\left(x_{0}\right)=\beta\left(\epsilon, x_{0}\right)+\gamma\left(\epsilon, x_{0}\right),
$$

From Assumption 1 and 3 of Theorem 1, each iterated integral involved in (57) satisfies, in the neighborhood of $x_{0}=0$,

$$
\left|\int_{0}^{T} u_{I}\left(x_{0}\right)\right| \leq K\left|x_{0}\right|
$$

for some positive constant $K$. This implies, in particular, that

$$
\frac{\left|\beta\left(\epsilon, x_{0}\right)\right|}{\left|x_{0}\right|} \leq K \sum_{|I| \leq 1 / \alpha}\left|\left(d_{I}^{h_{\epsilon}} i d\right)\left(x_{0}\right)\right| .
$$

Recalling that each product $d_{I}^{h_{\epsilon}}$ contains at least one $h_{\epsilon, i}$ and that $h_{i}(0, x)=0, \forall x \in \mathbb{R}^{n}$, one deduces that every function $\left.\left(\epsilon, x_{0}\right) \mapsto d_{I}^{h_{\epsilon}} i d\right)\left(x_{0}\right)$ involved in right-hand side of the above inequality is continuous w.r.t $x_{0}$ and $\epsilon$, and vanishes at $\epsilon=0$. Therefore, for $\delta$ small enough, and using the fact that the number of multi-indices $I$ such that $|I|<1 / \alpha$ is finite

$$
\sup _{\left|x_{0}\right| \leq \delta} \frac{\left|\beta\left(\epsilon, x_{0}\right)\right|}{\left|x_{0}\right|} \longrightarrow 0 \text { as } \epsilon \longrightarrow 0 .
$$

This establishes (44). Let us now define

$$
\tilde{u}_{i}\left(x_{0}, t\right) \triangleq \frac{u_{i}\left(x_{0}, t\right)}{\left|x_{0}\right|^{\alpha-\sigma}} \quad(i=1, \ldots, m)
$$

with $\sigma>0$ so that, by Assumption 1 of Theorem 1, $\tilde{u}_{i}$ is continuous. Then,

$$
\gamma\left(\epsilon, x_{0}\right)=\sum_{|I|>1 / \alpha}\left|x_{0}\right|^{|I|(\alpha-\sigma)}\left(d_{I}^{h_{\epsilon}} i d\right)\left(x_{0}\right) \int_{0}^{T} \tilde{u}_{I}\left(x_{0}\right) .
$$


Choosing $\sigma$ small enough so that $|I|(\alpha-\sigma) \geq 1+\mu_{0}>1$ for every $I$ such that $|I|>1 / \alpha$, and since, from Proposition 1, the series

$$
\sum_{|I|>1 / \alpha}\left(d_{I}^{h_{\epsilon}} i d\right)\left(x_{0}\right) \int_{0}^{T} \tilde{u}_{I}\left(x_{0}\right)
$$

is uniformly absolutely convergent for $x_{0}$ small enough, one obtains (provided that $\left|x_{0}\right|<1$ )

$$
\left|\gamma\left(\epsilon, x_{0}\right)\right| \leq\left|x_{0}\right|^{1+\mu_{0}} S\left(\epsilon, x_{0}\right)
$$

with

$$
S\left(\epsilon, x_{0}\right) \triangleq \sum_{|I|>1 / \alpha}\left|\left(d_{I}^{h_{\epsilon}} i d\right)\left(x_{0}\right)\right|\left|\int_{0}^{T} \tilde{u}_{I}\left(x_{0}\right)\right|,
$$

a positive continuous function. Relation (45) directly follows from this inequality, and this concludes the proof of Lemma 1.

\section{Proof of Theorem 2}

We first start with the proof of i).

Verification of Assumption 1: It follows directly from the definition (25) of $u$ and from Step 7, which requires each function $v_{\tau_{i}^{k}}^{k, c}$ to be a $O\left(|x|^{\frac{1}{l(k)}}\right)$.

Verification of Assumption 2: Since every $\tau_{p}^{k}$ belongs to $\{1, \ldots, m\}$,

$$
\sum_{i=1}^{m} \sum_{p: \tau_{p}^{k}=i} f_{i} \alpha_{\tau_{p}^{k}}^{k, c} v_{\tau_{p}^{k}}^{k, c}=\sum_{s=1}^{l(k)} f_{\tau_{s}^{k}} \alpha_{\tau_{s}^{k}}^{k, c} v_{\tau_{s}^{k}}^{k, c}
$$

so that, by (25),

$$
\sum_{i=1}^{m} f_{i}(x) u_{i}\left(x_{0}, t\right)=\sum_{k=1}^{K} \sum_{c=1}^{C(k)} \sum_{s=1}^{l(k)} f_{\tau_{s}^{k}}(x) \alpha_{\tau_{s}^{k}}^{k, c}(t) v_{\tau_{s}^{k}}^{k, c}\left(x_{0}\right) .
$$

To simplify the notations, let us introduce the following onto map from $U \subset(\mathbb{N}-\{0\}) \times(\mathbb{N}-\{0\})$ to $V=$ $\left\{1, \ldots, \sum_{k=1}^{K} C(k)\right\}$

$$
\boldsymbol{q}:(k, c) \longmapsto q=c+\sum_{i=0}^{k-1} C(i) \quad(\text { with } C(0)=0),
$$

and the inverse map

$$
\boldsymbol{q}^{-1}: q \in\left\{\sum_{i=0}^{k-1} C(i)+1, \ldots, \sum_{i=0}^{k} C(i)\right\} \longmapsto(\boldsymbol{k}(q), \boldsymbol{c}(q)) \triangleq\left(k, q-\sum_{i=0}^{k-1} C(i)\right) .
$$

We can then rewrite (60) as

$$
\sum_{i=1}^{m} f_{i}(x) u_{i}\left(x_{0}, t\right)=\sum_{q=1}^{Q} \sum_{s=1}^{S(q)} Z_{s}^{q}(x) \gamma_{s}^{q}\left(x_{0}, t\right)
$$


with

$$
\begin{aligned}
\gamma_{s}^{q} & \triangleq \alpha_{s}^{q} v_{s}^{q} \\
\alpha_{s}^{q} & \triangleq \alpha_{\tau_{s}^{k(q)}}^{\boldsymbol{k}(q), \boldsymbol{c}(q)} \\
v_{s}^{q} & \triangleq v_{\tau_{s}^{\mathrm{k}(q)}}^{\boldsymbol{k}(q), \boldsymbol{c}(q)} \\
Z_{s}^{q} & \triangleq f_{\tau_{s}^{\mathrm{k}(q)}} \\
S(q) & \triangleq l(\boldsymbol{k}(q))) \\
Q & \triangleq \sum_{k=1}^{K} C(k) .
\end{aligned}
$$

As a consequence, the solution at time $T$ of $\dot{x}=\sum_{i=1}^{m} f_{i}(x) u_{i}\left(x_{0}, t\right)$, with $x(0)=x_{0}$, is given by

$$
\begin{aligned}
x(T)= & x_{0}+\sum_{i=1}^{+\infty} \sum_{q_{1}, \ldots, q_{i}=1}^{Q} \sum_{s_{1}=1}^{\left.S\left(q_{1}\right)\right)} \cdots \sum_{s_{i}=1}^{S\left(q_{i}\right)}\left(Z_{s_{1}}^{q_{1}} \ldots Z_{s_{i}}^{q_{i}} i d\right)\left(x_{0}\right) \int_{0}^{T} \int_{0}^{t_{i}} \ldots \int_{0}^{t_{2}} \gamma_{s_{i}}^{q_{i}}\left(x_{0}, t_{i}\right) \ldots \\
& \gamma_{s_{1}}^{q_{1}}\left(x_{0}, t_{1}\right) d t_{1} \ldots d t_{i} .
\end{aligned}
$$

Lemma 2. Consider $J\left(\leq \operatorname{Max}_{k \in\{1, \ldots, K\}} l(k)\right)$ functions $\alpha_{j}(j \in\{1, \ldots, J\})$ taken in the set of trigonometric functions $\alpha_{\tau_{i}^{k}}^{k, c}$ defined by (22), with $l(k) \geq 2$.

Let $\omega_{j}$ denote the frequency associated with the function $\alpha_{j}$, and define the function $F_{\alpha_{1}, \ldots, \alpha_{J}}:[0, T] \rightarrow \mathbb{R}$ as follows

$$
F_{\alpha_{1}, \ldots, \alpha_{J}}(t)=\int_{0}^{t} \int_{0}^{t_{J}} \ldots \int_{0}^{t_{2}} \alpha_{J}\left(t_{J}\right) \ldots \alpha_{1}\left(t_{1}\right) d t_{1} \ldots d t_{J}
$$

If, for each M.C. set $\Omega^{k, c}$, the set $\left\{\omega_{1}, \ldots, \omega_{J}\right\}$ contains at most $l(k)$ elements which belong to $\Omega^{k, c}$, without containing $\Omega^{k, c}$ itself, then $F_{\alpha_{1}, \ldots, \alpha_{J}}(T)=0$.

The above technical result is just needed to establish the following lemma.

Lemma 3. The iterated integral

$$
\int_{0}^{T} \int_{0}^{t_{i}} \ldots \int_{0}^{t_{2}} \gamma_{s_{i}}^{q_{i}}\left(x_{0}, t_{i}\right) \ldots \gamma_{s_{1}}^{q_{1}}\left(x_{0}, t_{1}\right) d t_{1} \ldots d t_{i}
$$

is a $o\left(x_{0}\right)$ when some of the $q_{j}$ 's $(j \in\{1, \ldots, i\})$ are not equal, and when $q_{1}=q_{2}=\cdots=q_{i} \triangleq q$ and $\left(s_{1}, \ldots, s_{i}\right) \neq(\sigma(1), \ldots, \sigma(S(q))), \forall \sigma \in \mathfrak{S}(S(q))$.

The proofs of these lemmas are given at the end of this section.

In view of Lemma 3, using also the fact that each $v_{s_{i}}^{q_{i}}\left(x_{0}\right)$ is (by Step 7) a $o\left(\left|x_{0}\right|^{a}\right.$ ) for some positive $a$ so that one can infer uniform convergence arguments similar to these developped in the proof of Lemma 1 (65) 
simplifies to

$$
\begin{aligned}
& x(T)=x_{0}+\sum_{q=1}^{Q} \sum_{\sigma \in \mathfrak{S}(S(q))}\left(Z_{\sigma(1)}^{q} \ldots Z_{\sigma(S(q))}^{q} i d\right)\left(x_{0}\right) \int_{0}^{T} \int_{0}^{t_{S(q)}} \ldots \int_{0}^{t_{2}} \gamma_{\sigma(S(q))}^{q}\left(x_{0}, t_{S(q)}\right) \ldots \\
& \ldots \gamma_{\sigma(1)}^{q}\left(x_{0}, t_{1}\right) d t_{1} \ldots d t_{S(q)}+o\left(x_{0}\right) \\
&= x_{0}+\sum_{k=1}^{K} \sum_{c=1}^{C(j)} \sum_{\substack{\sigma \in\left(\tau_{\sigma(1)}^{k}, \ldots, \tau_{\sigma(l(k))}^{k}\right) \\
\sigma(l(k))}}^{\left(f_{I} i d\right)\left(x_{0}\right) \int_{0}^{T} \alpha_{I}^{k, c} v_{I}^{k, c}\left(x_{0}\right)+o\left(x_{0}\right)} \\
&=x_{0}+T \tilde{F}\left(x_{0}\right) a\left(x_{0}\right)+o\left(x_{0}\right) \\
&=\left(I_{n}+\tilde{F}(0) G\right) x_{0}+o\left(x_{0}\right),
\end{aligned}
$$

where the second and third equalities come from (64) and (33), and the fourth one from (20). Assumption 2 is thus verified by construction of $G$ in Step 2 .

Verification of Assumption 3: From (25) and (64),

$$
u_{i}(x, t)=\sum_{q=1}^{Q} \sum_{s: \tau_{s}^{k}(q)}=i
$$

Therefore, for any $I, \int_{0}^{T} u_{I}(x)$ is a sum of terms of the form

$$
\int_{0}^{T} \int_{0}^{t_{|I|}} \ldots \int_{0}^{t_{2}} \gamma_{s_{|I|}}^{q_{|I|}}\left(x, t_{|I|}\right) \ldots \gamma_{s_{1}}^{q_{1}}\left(x, t_{1}\right) d t_{1} \ldots d t_{|I|},
$$

for some multi-indices $\left(q_{1}, \ldots, q_{|I|}\right)$, and $\left(s_{1}, \ldots, s_{|I|}\right)$. From Lemma 3 , we only need to consider the case where $q_{1}=\ldots=q_{|I|} \triangleq q$ and $\left(s_{1}, \ldots, s_{|I|}\right)=(\sigma(1), \ldots, \sigma(|I|))$ with $|I|=S(q)$, since otherwise the expression in (69) is known to be a $o(x)$. In this case (69) rewrites as

$$
\begin{aligned}
& \prod_{i=1}^{S(q)} v_{i}^{q}(x) \int_{0}^{T} \int_{0}^{t_{S(q)}} \ldots \int_{0}^{t_{2}} \alpha_{S_{S(q)}}^{q} \ldots \alpha_{s_{1}}^{q} d t_{1} \ldots d t_{S(q)} \\
& =\sum_{j: \mathcal{B}_{j} \in P_{\mathrm{k}(q)}} \mu_{j}^{q} a_{j}(x) \int_{0}^{T} \int_{0}^{t_{S(q)}} \ldots \int_{0}^{t_{2}} \alpha_{S_{S(q)}}^{q} \ldots \alpha_{s_{1}}^{q} d t_{1} \ldots d t_{S(q)} .
\end{aligned}
$$

Since $a$ is a linear map, each iterated integral is a $O(x)$ so that Assumption 3 is verified. This concludes the proof of part $i$ ).

Finally, the proof of $i i)$ comes from that, in the f.l.a. case (33) is also true for the perturbed system $\left(S_{\epsilon}\right)$. Therefore, repeating the proof above with the perturbed system (68) becomes

$$
\begin{aligned}
x(T) & =x_{0}+\sum_{k=1}^{K} \sum_{c=1}^{C(j)} \sum_{\substack{\left(\tau_{\sigma(1)}^{k}, \ldots, \tau_{\sigma(l(k))}^{k} \\
\sigma \in \mathfrak{S}(l(k))\right.}}\left((f+h(\epsilon, .))_{I} i d\right)\left(x_{0}\right) \int_{0}^{T} \alpha_{I}^{k, c} v_{I}^{k, c}\left(x_{0}\right)+o\left(x_{0}\right) \\
& =x_{0}+T \tilde{F}_{\epsilon}\left(x_{0}\right) a\left(x_{0}\right)+o\left(x_{0}\right) \\
& =\left(I_{n}+\tilde{F}_{\epsilon}(0) G\right) x_{0}+o\left(x_{0}\right),
\end{aligned}
$$


and ii) follows. There only remains to prove Lemmas 2 and 3.

Proof of Lemma 2. Each sine or cosine function $\alpha_{j}$ is first decomposed into the sum of two exponential functions

$$
\alpha_{j}(t)=\eta_{j}^{1} e^{i \omega_{j} t}+\eta_{j}^{-1} e^{-i \omega_{j} t}
$$

For instance, if $\alpha_{j}(t)=\cos \left(\omega_{j} t\right)$ then $\eta_{j}^{1}=\eta_{j}^{-1}=\frac{1}{2}$, and if $\alpha_{j}(t)=\sin \left(\omega_{j} t\right)$ then $\eta_{j}^{1}=-\eta_{j}^{-1}=\frac{1}{2 i}$. Note that in any case we have $\left(\eta_{j}^{1}\right)^{2}=\left(\eta_{j}^{-1}\right)^{2}$. The proof of Lemma 2 is based on the following claim.

Claim 1. Let $\left(s_{J}, \ldots, s_{J-r}\right)$, with $r \in\{1, \ldots, J-1\}$, denote an element in the set $S_{r+1}=\{-1,1\}^{r+1}$, if $\sum_{j=0}^{r} s_{J-j} \omega_{J-j}=0$ then $\prod_{j=0}^{r} \eta_{J-j}^{s_{J-j}}+(-1)^{r} \prod_{j=0}^{r} \eta_{J-j}^{-s_{J-j}}=0$.

In order to prove this claim, let $\left\{\omega_{l_{m}}\right\}$, with $l_{m} \in\{J-r, \ldots, J\}$ and $m \in\{0, \ldots, \bar{r}(\leq r)\}$, denote the set of different frequencies involved in the set $\left\{\omega_{J-r}, \ldots, \omega_{J}\right\}$, and define $p_{l_{m}}$ as the number of frequencies in $\left\{\omega_{J-r}, \ldots, \omega_{J}\right\}$ equal to $\omega_{l_{m}}\left(\Rightarrow \sum_{m=0}^{\bar{r}} p_{l_{m}}=r+1\right)$. Then

$$
\sum_{j=0}^{r} s_{J-j} \omega_{J-j}=\sum_{m=0}^{\bar{r}} \lambda_{l_{m}} \omega_{l_{m}}
$$

with $\lambda_{l_{m}}=\sum_{j: \omega_{J-j}=\omega_{l_{m}}} s_{J-j}$, and also, since $s_{J-j}= \pm 1$

$$
\sum_{m}\left|\lambda_{l_{m}}\right| \leq \sum_{m} p_{l_{m}} \leq J \leq \operatorname{Max}_{k \in\{1, \ldots, K\}} l(k)
$$

Moreover

$$
\prod_{j=0}^{r} \eta_{J-j}^{s_{J-j}}+(-1)^{r} \prod_{j=0}^{r} \eta_{J-j}^{-s_{J-j}}=\prod_{m=0}^{\bar{r}}\left(\eta_{l_{m}}^{s_{l_{m}}}\right)^{p_{l_{m}}}+(-1)^{\left(\sum_{m=0}^{\bar{r}} p_{l_{m}}-1\right)} \prod_{m=0}^{\bar{r}}\left(\eta_{l_{m}}^{-s_{l_{m}}}\right)^{p_{l_{m}}}
$$

Now, since $\sum_{m}\left|\lambda_{l_{m}}\right| \leq \operatorname{Max} x_{k \in\{1, \ldots, K\}} l(k)$, one deduces from the assumption in Lemma 2 and by using the independence of the M.C. sets $\Omega^{k, c}$, that the equality $\sum_{m=0}^{\bar{r}} \lambda_{l_{m}} \omega_{l_{m}}=0$ implies that $\lambda_{l_{m}}=0, \forall m \in\{0, \ldots, \bar{r}\}$. Indeed, if some $\lambda_{l_{m}}$ were different of zero then the set $\left\{\omega_{J-r}, \ldots, \omega_{J}\right\}$ would have to contain either the M.C. set $\Omega^{k, c}$ to which $\omega_{l_{m}}$ belongs, or more than $l(k)$ terms belonging to $\Omega^{k, c}$. The fact that $\lambda_{l_{m}}=0$ in turn implies that the number $p_{l_{m}}$ of frequencies equal to $\omega_{l_{m}}$ is even. Therefore, $\left(\eta_{l_{m}}^{s_{l_{m}}}\right)^{p_{l_{m}}}=\left(\eta_{l_{m}}^{-s_{l_{m}}}\right)^{p_{l_{m}}}, \sum_{m=0}^{\bar{r}} p_{l_{m}}$ is even, and $(-1)\left(\sum_{m=0}^{\bar{r}} p_{l_{m}}-1\right)=-1$. It is then clear that the right member of $(72)$ is equal to zero.

Having proved Claim 1, let us return to the proof of Lemma 2. Clearly, $F_{\alpha_{1}}(T)=0$. Let us proceed by induction and show that if $F_{\alpha_{1}, \ldots, \alpha_{j}}(T)=0$, for $j \in\{1, \ldots, J-1\}$ then $F_{\alpha_{1}, \ldots, \alpha_{J}}(T)=0$. By definition of the function $F_{\alpha_{1}, \ldots, \alpha_{J}}$

$$
\begin{aligned}
F_{\alpha_{1}, \ldots, \alpha_{J}}(T) & =\int_{0}^{T} \alpha_{J}(t) F_{\alpha_{1}, \ldots, \alpha_{J-1}}(t) d t \\
& =\int_{0}^{T}\left(\sum_{s_{J} \in S_{1}} \eta_{J}^{s_{J}} e^{i s_{J} \omega_{J} t}\right) F_{\alpha_{1}, \ldots, \alpha_{J-1}}(t) d t .
\end{aligned}
$$


Integration by parts of the above integral, using the assumption that $F_{\alpha_{1}, \ldots, \alpha_{J-1}}(T)=0$ and the fact that $\frac{d}{d t} F_{\alpha_{1}, \ldots, \alpha_{J-1}}=\alpha_{J-1}(t) F_{\alpha_{1}, \ldots, \alpha_{J-2}}$, yields

$$
F_{\alpha_{1}, \ldots, \alpha_{J}}(T)=-\int_{0}^{T}\left(\sum_{\left(s_{J}, s_{J-1}\right) \in S_{2}} \frac{\eta_{J}^{s_{J}} \eta_{J-1}^{s_{J-1}} e^{i\left(s_{J} \omega_{J}+s_{J-1} \omega_{J-1}\right) t}}{i s_{J} \omega_{J}}\right) F_{\alpha_{1}, \ldots, \alpha_{J-2}}(t) d t .
$$

Using Claim 1 and the symmetry of $S_{2}$, the above equality may also be written as

$$
F_{\alpha_{1}, \ldots, \alpha_{J}}(T)=-\int_{0}^{T}\left(\sum_{\substack{\left(s_{J}, s_{J-1}\right) \in S_{2} \\ s_{J} \omega_{J}+s_{J-1} \omega_{J-1} \neq 0}} \frac{\eta_{J}^{s_{J}} \eta_{J-1}^{s_{J-1}} e^{i\left(s_{J} \omega_{J}+s_{J-1} \omega_{J-1}\right) t}}{i s_{J} \omega_{J}}\right) F_{\alpha_{1}, \ldots, \alpha_{J-2}}(t) d t .
$$

This process of integration by parts can be continued until the integrand in the right member of the equality does not contain any function $F_{\alpha_{1}, \ldots, \alpha_{j}}$. Each time Claim 1 and the symmetry of the sets $S_{j}$ are invoked to discard the contribution of frequency combinations which are equal to zero. We finally obtain in this way

$$
\begin{gathered}
F_{\alpha_{1}, \ldots, \alpha_{J}}(T)=(-1)^{J-1} \int_{0}^{T} \underset{\left(s_{J}, \ldots, s_{1}\right) \in S_{J}}{\sum_{j=0}^{r} s_{J-r} \omega_{J-r} \neq 0, r \in\{0, \ldots, J-1\}} \\
i^{J-1} \prod_{r=0}^{J-2}\left(\sum_{j=0}^{r} s_{J-j} \omega_{J-j}\right)
\end{gathered}
$$

Since the integrand is the sum of terms whose integrals over $[0, T]$ are clearly equal to zero, we thus have shown that $F_{\omega_{1}, \ldots, \omega_{J}}(T)=0$, and the proof is complete.

Proof of Lemma 3. From the definition of $\gamma_{s}^{q}$ in (64), the iterated integral (67) may also be written as

$$
v_{s_{i}}^{q_{i}}\left(x_{0}\right) \ldots v_{s_{1}}^{q_{1}}\left(x_{0}\right) \int_{0}^{T} \int_{0}^{t_{i}} \ldots \int_{0}^{t_{2}} \alpha_{s_{i}}^{q_{i}}\left(t_{i}\right) \ldots \alpha_{s_{1}}^{q_{1}}\left(t_{1}\right) d t_{1} \ldots d t_{i}
$$

Let us first consider the situation when some of the $q_{j}$ 's $(j \in\{1, \ldots, i\})$ are not equal. Then the set $\left\{\omega_{s_{1}}^{q_{1}}, \ldots, \omega_{s_{i}}^{q_{i}}\right\}$ either contains for each $(k, c)$ less than $l(k)$ terms belonging to $\Omega^{k, c}$, or contains for some $(k, c)$ at least $l(k)$ terms belonging to $\Omega^{k, c}$. In the first case, the integral (73) is equal to zero by application of Lemma 2 , so that it is also a $o\left(x_{0}\right)$. In the second case, the set $\left\{v_{s_{i}}^{q_{i}}\left(x_{0}\right), \ldots, v_{s_{1}}^{q_{1}}\left(x_{0}\right)\right\}$ contains $l(k)$ terms, each of them a $O\left(\left|x_{0}\right|^{\frac{1}{(k)}}\right)$ as imposed in Step 7 of the algorithm, plus additional terms (at least one), each of them a $O\left(\left|x_{0}\right|^{\beta}\right)$ for some $\beta>0$. Therefore, the product $v_{s_{i}}^{q_{i}}\left(x_{0}\right) \ldots v_{s_{1}}^{q_{1}}\left(x_{0}\right)$ is again a $o\left(x_{0}\right)$.

Let us now consider the case when all $q_{j}$ 's are equal (to $\left.q\right)$ and $\left(s_{1}, \ldots, s_{i}\right) \neq(\sigma(1), \ldots, \sigma(S(q)), \forall \sigma \in$ $\mathfrak{S}(S(q))$. The equality of the $q_{j}$ 's implies that all frequencies $\omega_{s_{j}}^{q}$ belong to a unique M.C. set $\Omega^{k, c}$. The latter inequality implies that the set $\left\{\omega_{s_{1}}^{q}, \ldots, \omega_{s_{i}}^{q}\right\}$ does not coincide with $\Omega^{k, c}$. Therefore, if $i \leq l(k)$, then this set does not contain $\Omega^{k, c}$ and, by application of Lemma 2, the iterated integral in (73) is zero. If $i>l(k)$, then the product $v_{s_{i}}^{q}\left(x_{0}\right) \ldots v_{s_{1}}^{q}\left(x_{0}\right)$ is, in view of the condition imposed in Step 7 of the algorithm, a $O\left(\left|x_{0}\right|^{\frac{i}{l(k)}}\right)$, and thus a $o\left(x_{0}\right)$. This concludes the proof of Lemma 3, and the proof of Theorem 2 is now complete.

\section{Proof of Theorem 3}

Assumptions 1 and 3 of Theorem 1 are obviously verified for the system (35), since they only involve the control law and do not depend on the control system. Hence, we only need to take care of Assumption 2 and 
show that the solution of

$$
\dot{z}=\sum_{i=1}^{m}\left(b_{i}(z)+g_{i}(z)\right) u_{i}\left(z_{0}, t\right), \quad z\left(t_{0}\right)=z_{0}
$$

satisfies

$$
z(T)=\bar{A} z_{0}+o\left(z_{0}\right)
$$

for some discrete-stable matrix $\bar{A}$. Let us first introduce some notations. Without loss of generality, we assume that the variables $z_{i}$ are ordered by increasing weight, i.e.

$$
r_{1} \leq r_{2} \leq \ldots \leq r_{n}
$$

and decompose $z$ as $z=\left(z^{1}, \ldots, z^{P}\right)$, where each $z^{p}(1 \leq p \leq P)$ is the sub-vector of $z$ whose components have same weight $r^{p}\left(r_{1} \leq r^{p} \leq r_{n}\right)$ with

$$
r_{1}=r^{1}<r^{2}<\ldots<r^{P}=r_{n}
$$

In a similar way, a map $f$ from $\mathbb{R}^{n}$ to $\mathbb{R}^{n}$ can be decomposed as $f=\left(f^{1}, \ldots, f^{P}\right)$.

We may now proceed with the proof. The solution of (74) can be expanded as

$$
z(t)=z_{0}+\sum_{I}\left((b+g)_{I} i d\right)\left(z_{0}\right) \int_{0}^{t} u_{I}\left(z_{0}\right) .
$$

By Proposition 1, the series in the right-hand side of this equality is uniformly convergent w.r.t. $z_{0}\left(\left|z_{0}\right| \leq \delta\right)$ and $t \in[0, T]$. The above expression may be rewritten as

$$
z(t)=z_{0}+\sum_{I}\left(b_{I} i d\right)\left(z_{0}\right) \int_{0}^{t} u_{I}\left(z_{0}\right)+\sum_{I}\left(d_{I}^{g} i d\right)\left(z_{0}\right) \int_{0}^{t} u_{I}\left(z_{0}\right) .
$$

Here, $d_{I}^{g} \triangleq d_{i_{1}}^{g} \cdots d_{i_{k}}^{g}$ (for $\left.I=\left(i_{1}, \ldots, i_{k}\right)\right)$, where each $d_{i}^{g}$ belongs to $\left\{b_{i}, g_{i}\right\}$, and the product $d_{I}^{g}$ contains at least one of the $g_{i}$ 's. Note that the first sum in the right-hand side involves a finite number of terms because $\left\{b_{1}, \ldots, b_{m}\right\}$ is a nilpotent set of vector fields. As a consequence, the series defined by the second sum is uniformly convergent w.r.t. $z_{0}$ and $t$. Each of these two sums is now considered separately.

Since Assumption 2 in Theorem 1 is verified for the approximating system (34), we have

$$
z_{0}+\sum_{I}\left(b_{I} i d\right)\left(z_{0}\right) \int_{0}^{T} u_{I}\left(z_{0}\right)=A z_{0}+o\left(z_{0}\right) .
$$

where $A$ is a discrete-stable matrix. We claim that the matrix $A$ is necessarily block upper-triangular in the sense that

$$
A z_{0}=\left(\begin{array}{ccc}
A_{11} & \star & \star \\
& \ddots & \star \\
0 & & A_{P P}
\end{array}\right)\left(\begin{array}{c}
z_{0}^{1} \\
\vdots \\
z_{0}^{P}
\end{array}\right) .
$$

In order to prove this assertion, it is clearly sufficient to show that, for $p=1, \ldots, P$,

$$
\sum_{I}\left(b_{I} i d\right)^{p}\left(z_{0}\right) \int_{0}^{T} u_{I}\left(z_{0}\right)=\sum_{q \geq p} \tilde{A}_{p, q} z_{0}^{q}+o\left(z_{0}\right)
$$


for some matrices $\tilde{A}_{p, q}$. Let us rewrite the sum in the left-hand side of (79) as

$$
\sum_{\|I\|<r^{p},|I| \leq \frac{1}{\alpha}}\left(b_{I} i d\right)^{p} \int_{0}^{T} u_{I}+\sum_{\|I\| \geq r^{p},|I| \leq \frac{1}{\alpha}}\left(b_{I} i d\right)^{p} \int_{0}^{T} u_{I}+\sum_{|I|>\frac{1}{\alpha}}\left(b_{I} i d\right)^{p} \int_{0}^{T} u_{I}
$$

where the argument $z_{0}$ is omitted for the sake of conciseness.

We first note that the last sum in (80) is a $o\left(z_{0}\right)$ because, from Assumption 1, each $\int_{0}^{T} u_{I}$ is itself a $o\left(z_{0}\right)$ when $|I|>1 / \alpha$.

From Assumption 3-bis in Theorem 3, all iterated integrals in the second sum are of the form

$$
\sum_{k: r_{k} \geq r^{p}} a_{I, k} z_{0, k}+o\left(z_{0}\right)
$$

which may also be written as

$$
\sum_{p \leq q \leq P} \tilde{a}_{I, q} z_{0}^{q}+o\left(z_{0}\right)
$$

since any $z_{0, k}$ whose weight $r_{k}$ is greater or equal to $r^{p}$ has to be an element of some $z_{0}^{q}$ with $q \geq p$. This clearly implies that the second sum in (80) can be written as the right-hand side of (79).

Let us finally consider the first sum in $(80)$. Since $\left(b_{I} i d\right)^{p}\left(z_{0}\right)$ is just $b_{I} z^{p}$ evaluated at $z_{0}$, and since each component of $z^{p}$ is homogeneous of degree $r^{p}$, it follows that each component of $\left(b_{I} i d\right)^{p}$ is a function homogeneous of positive degree $r^{p}-\|I\|$. Therefore, each $\left(b_{I} i d\right)^{p}$ vanishes at the origin and, since it is a smooth function, there exists $K_{I}>0$ such that $\left|\left(b_{I} i d\right)^{p}\left(z_{0}\right)\right| \leq K_{I}\left|z_{0}\right|$. This inequality, combined with Assumption 3 which tells us that $\int_{0}^{T} u_{I}\left(z_{0}\right)=O\left(z_{0}\right)$, implies that the first sum in (80) is a $o\left(z_{0}\right)$. Therefore, relation (79) holds for every $p=1, \ldots, P$ and, subsequently, the matrix $A$ is block upper-triangular. Moreover, $A$ being a discrete-stable matrix, each matrix $A_{p p}$ on the block diagonal is necessarily a discrete-stable matrix itself.

Let us now show that

$$
\sum_{I}\left(d_{I}^{g} i d\right)\left(z_{0}\right) \int_{0}^{T} u_{I}\left(z_{0}\right)=C z_{0}+o\left(z_{0}\right)
$$

where $C$ is a block upper-triangular matrix with zeroes on the block diagonal, i.e.

$$
C z_{0}=\left(\begin{array}{ccc}
0 & \star & \star \\
\vdots & \ddots & \star \\
0 & \cdots & 0
\end{array}\right)\left(\begin{array}{c}
z_{0}^{1} \\
\vdots \\
z_{0}^{P}
\end{array}\right)+o\left(z_{0}\right)
$$

To this purpose, we just need to show that, for $p=1, \ldots, P$,

$$
\sum_{I}\left(d_{I}^{g} i d\right)^{p}\left(z_{0}\right) \int_{0}^{T} u_{I}\left(z_{0}\right)=\sum_{q>p} C_{p, q} z_{0}^{q}+o\left(z_{0}\right)
$$

for some matrices $C_{p, q}$. Let us again decompose the sum in the left-hand side of (83) as

$$
\sum_{\|I\| \leq r^{p},|I| \leq 1 / \alpha}\left(d_{I}^{g} i d\right)^{p} \int_{0}^{T} u_{I}+\sum_{\|I\|>r^{p},|I| \leq 1 / \alpha}\left(d_{I}^{g} i d\right)^{p} \int_{0}^{T} u_{I}+\sum_{|I|>1 / \alpha}\left(d_{I}^{g} i d\right)^{p} \int_{0}^{T} u_{I} .
$$


We start with the third sum in (84), and define

$$
\tilde{u}_{i}\left(z_{0}, t\right) \triangleq \frac{u_{i}\left(z_{0}, t\right)}{\left|z_{0}\right|^{\alpha-\sigma}} \quad(i=1, \ldots, m)
$$

with $\sigma>0$ small enough so that, by Assumption 1 of Theorem 1, $\tilde{u}_{i}$ is continuous. Then,

$$
\sum_{|I|>1 / \alpha}\left(d_{I}^{g} i d\right)^{p}\left(z_{0}\right) \int_{0}^{T} u_{I}\left(z_{0}\right)=\sum_{|I|>1 / \alpha}\left|z_{0}\right|^{|I|(\alpha-\sigma)}\left(d_{I}^{g} i d\right)\left(z_{0}\right) \int_{0}^{T} \tilde{u}_{I}\left(z_{0}\right)
$$

Choosing $\sigma$ small enough such that the inequality $|I|(\alpha-\sigma) \geq 1+\mu_{0}>1$ holds for every $I$ such that $|I|>1 / \alpha$, and using the fact that, from Proposition 1, the series

$$
\sum_{|I|>1 / \alpha}\left(d_{I}^{g} i d\right)\left(z_{0}\right) \int_{0}^{T} \tilde{u}_{I}\left(z_{0}\right)
$$

is uniformly absolutely convergent for $z_{0}$ small enough, we obtain (provided that $\left|z_{0}\right|<1$ )

$$
\left|\sum_{|I|>1 / \alpha}\left(d_{I}^{g} i d\right)^{p}\left(z_{0}\right) \int_{0}^{T} u_{I}\left(z_{0}\right)\right| \leq\left|z_{0}\right|^{1+\mu_{0}} S\left(z_{0}\right)
$$

with $S$ a continuous function. This establishes that the third sum in $(84)$ is a $o\left(z_{0}\right)$.

Let us now consider the second sum in (84). From Assumption 3-bis, and as pointed out before in the proof (with the only difference that $\|I\|$ is now taken strictly greater than $r^{p}$ ), all iterated integrals in this sum are of the form

$$
\sum_{q>p} \tilde{a}_{I, q} z_{0}^{q}+o\left(z_{0}\right)
$$

This implies that the second sum in (84) can be written as the right-hand side of (83).

Let us finally consider the first sum in (84). By definition of the product $d_{I}^{g}$, there is at least one term in this product which belongs to $\left\{g_{1}, \ldots, g_{m}\right\}$. Now, since $g_{i, j}=o\left(\rho^{r_{j}+d_{i}}\right)$, for $j=1, \ldots, n$ (relation (36)), the Taylor expansion of $g_{i}$ at the origin gives a sum of vector fields homogeneous of degree strictly larger than $d_{i}$. This in turn implies that each $d_{I}^{g}$ is a sum of differential operators of degree strictly larger than - $\|I\|$, and that every component of $\left(d_{I}^{g} i d\right)^{p}$ is a sum of homogeneous functions of degree strictly larger than $\left(r^{p}-\|I\|\right)$. Since $\left(r^{p}-\|I\|\right) \geq 0$, this degree is thus strictly positive. Therefore, every $\left(d_{I}^{g} i d\right)^{p}$ vanishes at the origin and, since it is also a smooth function, there exists $K_{I}>0$ such that $\left|\left(d_{I}^{g} i d\right)^{p}\left(z_{0}\right)\right| \leq K_{I}\left|z_{0}\right|$. This inequality, combined with Assumption 1 which implies that $\left|\int_{0}^{T} u_{I}\left(z_{0}\right)\right|$ tends to zero when $z_{0}$ tends to zero, implies that the first sum in $(84)$ is a $o\left(z_{0}\right)$. We have thus proved that $(83)$ holds for any $p=1, \ldots, P$, and, subsequently, that relations (81) and (82) also hold. It follows from (76), (77), and (81) that relation (75) is true with $\bar{A}=A+C$, a discrete-stable matrix. Therefore Assumption 2 is verified for the system (35), and this concludes the proof of Theorem 3.

\section{Proof of Theorem 4}

We give the proof for $\omega=1(\Rightarrow T=2 \pi)$-any other value of $\omega$ being taken care of by introducing the time-scaling $t \longmapsto \omega t$. Throughout the proof, the control vector fields associated with the chained system (39) 
are denoted as $b_{1}$ and $b_{2}$, i.e.

$$
\begin{aligned}
& b_{1}(x)=\left(1,0, x_{2}, \ldots, x_{n-1}\right)^{\prime} \\
& b_{2}(x)=(0,1,0, \ldots, 0)^{\prime} .
\end{aligned}
$$

For any $q>0$, and corresponding dilation $\delta^{r(q)}$ such that $r(q)=(1, q, q+1, \ldots, q+n-2)$, the v.f. $b_{1}$ and $b_{2}$ are $\delta^{r(q)}$-homogeneous of degree -1 and $-q$ respectively. In view of $(40), u_{1}(x, t)$ and $u_{2}(x, t)$ are $\delta^{r(q)}$ homogeneous of degree 1 and $q$ respectively. Therefore, since $q$ is positive, Assumption 1 of Theorem 1 is verified with $\alpha=\frac{1}{\max _{i} r_{i}(q)}=\frac{1}{q+n-2}$.

Let us now check that Assumption 2 is verified. The solution $x($.$) of (17)$ on the time-interval $[0, T]$ is given by

$$
x(t)=x_{0}+\sum_{I}\left(b_{I} i d\right)\left(x_{0}\right) \int_{0}^{t} u_{I}\left(x_{0}\right) \quad(t \in[0, T]) .
$$

It is simple to show by induction that

$$
(\underbrace{b_{1} \cdots b_{1}}_{k} i d)(x)=\left(0, \ldots, 0, x_{2}, \ldots, x_{n-k}\right)^{\prime} \quad(k \geq 2),
$$

which implies

$$
(b_{2} \underbrace{b_{1} \cdots b_{1}}_{k} i d)(x)=(\underbrace{0, \ldots, 0}_{k+1}, 1,0, \ldots, 0)^{\prime} \quad(k \geq 1),
$$

and, subsequently

$$
\left(b_{i} b_{2} b_{1} \cdots b_{1} i d\right) \equiv 0 \quad \forall i=1,2 \text {. }
$$

Hence, the only multi-indices for which $b_{I} i d$ is not identically zero are those of the form $I=(1, \ldots, 1)$ or $I=(2,1, \ldots, 1)$, and one obtains from $(86,87,88)$,

$$
\begin{aligned}
x(T) & =x_{0}+\sum_{k=1}^{n-2}(\underbrace{b_{1} \cdots b_{1}}_{k} i d)\left(x_{0}\right) \int_{0}^{T} u_{(1, \ldots, 1)}\left(x_{0}\right)+\sum_{k=0}^{n-2}(b_{2} \underbrace{b_{1} \cdots b_{1}}_{k} i d)\left(x_{0}\right) \int_{0}^{T} u_{(2,1, \ldots, 1)}\left(x_{0}\right) \\
& =x_{0}+b_{1}\left(x_{0}\right) \int_{0}^{T} u_{1}\left(x_{0}\right)+\sum_{k=0}^{n-2}(b_{2} \underbrace{b_{1} \cdots b_{1}}_{k} i d)\left(x_{0}\right) \int_{0}^{T} u_{(2,1, \ldots, 1)}\left(x_{0}\right)+o\left(x_{0}\right) \\
& =x_{0}+\left(\begin{array}{ccccc}
1 & 0 & 0 & \ldots & 0 \\
0 & 1 & 0 & \ldots & \vdots \\
x_{0,2} & 0 & 1 & & \vdots \\
\vdots & \vdots & \ddots & \ddots & \vdots \\
x_{0, n-2} & 0 & \ldots & 0 & 1
\end{array}\right)\left(\begin{array}{c}
\int_{0}^{T} u_{1}\left(x_{0}\right) \\
\int_{0}^{T} u_{2}\left(x_{0}\right) \\
\int_{0}^{T} u_{(2,1)}\left(x_{0}\right) \\
\vdots \\
\int_{0}^{T} u_{(2,1, \ldots, 1)}\left(x_{0}\right)
\end{array}\right)+o\left(x_{0}\right) .
\end{aligned}
$$


Therefore

$$
x(T)=x_{0}+\left(\begin{array}{c}
\int_{0}^{T} u_{1}\left(x_{0}\right) \\
\int_{0}^{T} u_{2}\left(x_{0}\right) \\
\int_{0}^{T} u_{(2,1)}\left(x_{0}\right) \\
\vdots \\
\int_{0}^{T} u_{(2,1, \ldots, 1)}\left(x_{0}\right)
\end{array}\right)+o\left(x_{0}\right)
$$

Let us now calculate the iterated integral involved in the right-hand side of equality (90). First, we have

$$
\int_{0}^{T} u_{1}\left(x_{0}\right)=\left(k_{1}-1\right) x_{0,1}, \quad \int_{0}^{T} u_{2}\left(x_{0}\right)=\left(k_{2}-1\right) x_{0,2} .
$$

Calculation of the other integrals makes use of the following lemma.

Lemma 4. For any $k$ and $i$ in $\mathbb{N}-\{0\}$, and any $p \in\{1, \ldots, k\}$,

$$
\begin{aligned}
& \int_{0}^{2 \pi} \int_{0}^{t_{k+1}} \cdots \int_{0}^{t_{2}} \sin t_{k+1} \cdots \sin t_{1} d t_{1} \cdots d t_{k+1}=0 \\
& \int_{0}^{2 \pi} \int_{0}^{t_{k+1}} \cdots \int_{0}^{t_{2}} \sin t_{k+1} \cdots \sin t_{p+1} \cos i t_{p} \sin t_{p-1} \cdots \sin t_{1} d t_{1} \cdots d t_{k+1}=\left\{\begin{array}{cc}
0 & \text { if } i>k \\
\frac{2 \pi}{2^{k} k !} & \text { if } i=k \text { and } p=1 .
\end{array}\right.
\end{aligned}
$$

The proof of this lemma is given at the end of this section.

From (40)

$$
\begin{gathered}
\int_{0}^{T} u(\underbrace{2,1, \ldots, 1}_{k+1})^{\left(x_{0}\right)=} \sum_{i=3}^{n} \frac{2^{i-2}(i-2) !}{T} \frac{\left(k_{i}-1\right) x_{0, i}}{\rho_{q}^{i-(k+2)}\left(x_{0}\right)} \int_{0}^{T} \int_{0}^{t_{k+1}} \cdots \int_{0}^{t_{2}} \sin t_{k+1} \cdots \\
\\
\sin t_{2} \cos (i-2) t_{1} d t_{1} \ldots d t_{k+1}+o\left(x_{0}\right) .
\end{gathered}
$$

Using the fact that $\rho_{q}(x)=O\left(|x|^{\frac{1}{q+n-2}}\right)$

$$
\begin{aligned}
\int_{0}^{T} u & (\underbrace{2,1, \ldots, 1}_{k+1})^{\left(x_{0}\right)=} \sum_{i=k+2}^{n} \frac{2^{i-2}(i-2) !}{T} \frac{\left(k_{i}-1\right) x_{0, i}}{\rho_{q}^{i-(k+2)}\left(x_{0}\right)} \int_{0}^{T} \int_{0}^{t_{k+1}} \cdots \int_{0}^{t_{2}} \sin t_{k+1} \cdots \\
& \sin t_{2} \cos (i-2) t_{1} d t_{1} \ldots d t_{k+1}+o\left(x_{0}\right)
\end{aligned}
$$

and, by application of Lemma 4,

$$
\int_{0}^{T} u(\underbrace{2,1, \ldots, 1}_{k+1})^{\left(x_{0}\right)=\left(k_{k+2}-1\right) x_{0, k+2}+o\left(x_{0}\right) .}
$$

Using (91) and (93) in (90), we obtain

$$
x(T)=A x_{0}+o\left(x_{0}\right)
$$


with $A=\operatorname{diag}\left\{k_{1}, k_{2}, \ldots, k_{n}\right\}$, a discrete-stable matrix since $k_{i} \in(-1,1)(i=1, \ldots, n)$.

There remains to show that Assumptions 3 of Theorem 1 and 3-bis of Theorem 3 are verified. It is in fact sufficient to show that the stronger latter assumption holds. To this purpose, we must show that (38) holds for every possible $I$ (recall that $d_{1}=\operatorname{deg}\left(b_{1}\right)=-1$, and $d_{2}=\operatorname{deg}\left(b_{2}\right)=-q$ ).

Let us first consider the case where $I=(1, \ldots, 1)$. If $|I|=1$, then

$$
\int_{0}^{T} u_{I}(x)=\int_{0}^{T} u_{1}(x)=\left(k_{1}-1\right) x_{1}
$$

and (38) obviously holds. If $|I|>1$, then

$$
\begin{aligned}
\int_{0}^{T} u_{I}(x) & =\rho_{q}^{|I|}(x) \int_{0}^{T} \int_{0}^{t_{|I|}} \ldots \int_{0}^{t_{2}} \sin t_{|I|} \ldots \sin t_{1} d t_{|I|} \ldots d t_{1}+o(x) \\
& =o(x),
\end{aligned}
$$

where the last equality results from Lemma 4. Therefore, (38) holds for every $I$ which does not contain the index 2 .

Assume now that $I$ contains the index 2 twice at least. If $I=(2,2)$, then a simple calculation yields

$$
\int_{0}^{T} u_{I}(x)=o(x)
$$

If $|I|>2$, the iterated integral is $\delta^{r(q)}$-homogeneous of degree strictly larger than $2 q$. Since $q \geq n-2$ (see (41)) and $r_{i}(q) \leq q+n-2(i=1, \ldots, n)$, this degree is larger than the degree of homogeneity of each $x_{i}$, so that (95) also holds in this case.

Let us finally consider the case where $I$ contains the index 2 exactly once.

If $I=(2,1, \ldots, 1)$, the satisfaction of (38) follows from (93).

If the index 2 is not in the first entry, i.e. $I$ is of the form

$$
(\underbrace{1, \ldots, 1}_{p}, 2, \underbrace{1, \ldots, 1}_{k-p}) \quad(p>0),
$$

then $\|I\|=k+q$ and

$$
\begin{aligned}
& \int_{0}^{T} u_{I}(x)=\sum_{i=3}^{n} \frac{2^{i-2}(i-2) !}{T} \frac{\left(k_{i}-1\right) x_{i}}{\rho_{q}^{i-(k+2)}(x)} \int_{0}^{T} \int_{0}^{t_{k+1}} \ldots \int_{0}^{t_{2}} \sin t_{k+1} \ldots \sin t_{p+2} \cos (i-2) t_{p+1} \\
&=\sum_{i=k+2}^{n} \frac{2^{i-2}(i-2) !}{T} \frac{\left(k_{i}-1\right) x_{i}}{\rho_{q}^{i-(k+2)}} \int_{0}^{T} \int_{0}^{t_{k+1}} \ldots \int_{0}^{t_{2}} \sin t_{p} \ldots \sin t_{1} d t_{k+1} \ldots d t_{1}+o(x) \\
&=\quad\left(k_{k+2}-1\right) x_{k+2} \int_{0}^{T} \int_{0}^{t_{k+1}} \ldots \int_{0}^{t_{2}} \sin t_{p+2} \sin t_{p} \ldots \sin t_{1} d t_{k+1} \ldots d t_{1}+o(x) \\
& \sin t_{p+1} \ldots \sin t_{p+2} \cos (i-2) t_{p+1} \\
&+\sum_{i=k+3}^{n} \frac{2^{i-2}(i-2) !}{T} \frac{\left(k_{i}-1\right) x_{i}}{\rho_{q}^{i-(k+2)}(x)} \int_{0}^{T} \int_{0}^{t_{k+1}} \ldots \int_{0}^{t_{2}} \sin t_{k+1} \ldots \sin t_{p+2} \cos (i-2) t_{p+1} \\
& \sin t_{p} \ldots \sin t_{1} d t_{k+1} \ldots d t_{1}+o(x) .
\end{aligned}
$$


Since the weight of $x_{k+2}$ is equal to $k+q(=\|I\|)$, the first term in the right-hand side of the last equality is one of the linear terms involved in the right-hand side of relation (38), whereas all other iterated integrals are equal to zero by application of Lemma 4.

We have thus shown that relation (38) holds for every possible $I$, and this concludes the proof of Theorem 4. There only remains to prove Lemma 4.

Proof of Lemma 4. We shall use the following relation, the proof of which is easily worked out by induction on $k$ :

$$
\int_{0}^{t} \int_{0}^{t_{k}} \ldots \int_{0}^{t_{2}} \sin t_{k} \ldots \sin t_{1} d t_{1} \ldots d t_{k}=\sum_{j=1}^{k} c_{k, j}(1-\cos j t), \quad c_{k, k}=\frac{(-1)^{k-1}}{2^{k-1} k !}
$$

From this, we readily obtain the first equation in (92). We also deduce that (recall that $T=2 \pi$ ),

$$
\int_{0}^{T} \int_{0}^{t_{k+1}} \ldots \int_{0}^{t_{2}} \cos i t_{k+1} \sin t_{k} \ldots \sin t_{1} d t_{1} \ldots d t_{k+1}=\left\{\begin{array}{cl}
0 & \text { if } i>k \\
\frac{(-1)^{k}}{2^{k} k !} T & \text { if } i=k
\end{array}\right.
$$

Now, we claim that for any $i \geq k$,

$$
\begin{gathered}
\int_{0}^{T} \int_{0}^{t_{k+1}} \ldots \int_{0}^{t_{2}} \sin t_{k+1} \ldots \sin t_{2} \cos i t_{1} d t_{1} \ldots d t_{k+1} \\
=(-1)^{k} \int_{0}^{T} \int_{0}^{t_{k+1}} \ldots \int_{0}^{t_{2}} \cos i t_{k+1} \sin t_{k} \ldots \sin t_{1} d t_{1} \ldots d t_{k+1} .
\end{gathered}
$$

To show this, we view the first integral as a multiple integral on $\mathbb{R}^{k+1}$ (on the domain $\left\{\left(t_{1}, \ldots, t_{k+1}\right) \in \mathbb{R}^{k+1}: 0 \leq t_{1} \leq \ldots \leq t_{k+1} \leq T\right\}$, to be more precise) so that this integral can also be written as

$$
\int_{0}^{T} \int_{t_{1}}^{T} \ldots \int_{t_{k}}^{T} \cos i t_{1} \sin t_{2} \ldots \sin t_{k+1} d t_{1} \ldots d t_{k+1}
$$

Setting $\tau_{i} \triangleq t_{k+2-i}(i=1, \ldots, k+1)$, this gives

$$
\begin{gathered}
\int_{0}^{T} \int_{\tau_{k+1}}^{T} \ldots \int_{\tau_{2}}^{T} \cos i \tau_{k+1} \sin \tau_{k} \ldots \sin \tau_{1} d \tau_{1} \ldots d \tau_{k+1} \\
=\int_{0}^{T}\left(\int_{0}^{T}-\int_{0}^{\tau_{k+1}}\right) \ldots\left(\int_{0}^{T}-\int_{0}^{\tau_{2}}\right) \cos i \tau_{k+1} \sin \tau_{k} \ldots \sin \tau_{1} d \tau_{1} \ldots d \tau_{k+1} .
\end{gathered}
$$

Using (96), this last term simplifies into

$$
(-1)^{k} \int_{0}^{T} \int_{0}^{\tau_{k+1}} \ldots \int_{0}^{\tau_{2}} \cos i \tau_{k+1} \sin \tau_{k} \ldots \sin \tau_{1} d \tau_{1} \ldots d \tau_{k+1}
$$

and (98) follows. Finally (92) follows directly from (97) and (98). The proof of this lemma is now complete.

\section{REFERENCES}

[1] M.K. Bennani and P. Rouchon, Robust stabilization of flat and chained systems, in European Control Conference (ECC) (1995) 2642-2646. 
[2] R.W. Brockett, Asymptotic stability and feedback stabilization, Differential Geometric Control Theory, R.S. Millman R.W. Brockett and H.H. Sussmann Eds., Birkauser (1983).

[3] C. Canudas de Wit and O. J. Sørdalen, Exponential stabilization of mobile robots with nonholonomic constraints. IEEE Trans. Automat. Control 37 (1992) 1791-1797.

[4] M. Fliess, J. Lévine, P. Martin and P. Rouchon, Flatness and defect of non-linear systems: introductory theory and examples. Internat. J. Control 61 (1995) 1327-1361.

[5] H. Hermes, Nilpotent and high-order approximations of vector field systems. SIAM Rev. 33 (1991) $238-264$.

[6] A. Isidori, Nonlinear control systems. Springer Verlag, third edition (1995).

[7] M. Kawski, Geometric homogeneity and stabilization, in IFAC Nonlinear Control Systems Design Symp. (NOLCOS) (1995) 164-169.

[8] I. Kolmanovsky and N.H. McClamroch, Developments in nonholonomic control problems. IEEE Control Systems (1995) 20-36.

[9] J. Kurzweil and J. Jarnik, Iterated lie brackets in limit processes in ordinary differential equations. Results in Mathematics 14 (1988) 125-137.

[10] Z. Li and J.F. Canny, Nonholonomic motion planning. Kluwer Academic Press (1993).

[11] W. Liu, An approximation algorithm for nonholonomic systems. SIAM J. Contr. Opt. 35 (1997) 1328-1365.

[12] D.A. Lizárraga, P. Morin and C. Samson, Non-robustness of continuous homogeneous stabilizers for affine systems. Technical Report 3508, INRIA (1998). Available at http://www.inria.fr/RRRT/RR-3508.html

[13] R.T. M'Closkey and R.M. Murray, Exponential stabilization of driftless nonlinear control systems using homogeneous feedback. IEEE Trans. Automat. Contr. 42 (1997) 614-628.

[14] S. Monaco and D. Normand-Cyrot, An introduction to motion planning using multirate digital control, in IEEE Conf. on Decision and Control (CDC) (1991) 1780-1785.

[15] P. Morin, J.-B. Pomet and C. Samson, Design of homogeneous time-varying stabilizing control laws for driftless controllable systems via oscillatory approximation of lie brackets in closed-loop. SIAM J. Contr. Opt. (to appear).

[16] P. Morin, J.-B. Pomet and C. Samson, Developments in time-varying feedback stabilization of nonlinear systems, in IFAC Nonlinear Control Systems Design Symp. (NOLCOS) (1998) 587-594.

[17] P. Morin and C. Samson, Exponential stabilization of nonlinear driftless systems with robustness to unmodeled dynamics. Technical Report 3477, INRIA (1998).

[18] R.M. Murray and S.S. Sastry, Nonholonomic motion planning: Steering using sinusoids. IEEE Trans. Automat. Contr. 38 (1993) $700-716$.

[19] L. Rosier, Étude de quelques problèmes de stabilisation. PhD thesis, École Normale de Cachan (1993).

[20] C. Samson, Velocity and torque feedback control of a nonholonomic cart, in Int. Workshop in Adaptative and Nonlinear Control: Issues in Robotics. LNCIS, Vol. 162, Springer Verlag, 1991 (1990).

[21] O.J. Sørdalen and O. Egeland, Exponential stabilization of nonholonomic chained systems. IEEE Trans. Automat. Contr. 40 (1995) 35-49.

[22] G. Stefani, Polynomial approximations to control systems and local controllability, in IEEE Conf. on Decision and Control $(C D C)(1985) 33-38$

[23] G. Stefani, On the local controllability of scalar-input control systems, in Theory and Applications of Nonlinear Control Systems, Proc. of MTNS'84, C.I. Byrnes and A. Linsquist Eds., North-Holland (1986) 167-179.

[24] H.J. Sussmann and W. Liu, Limits of highly oscillatory controls ans approximation of general paths by admissible trajectories, in IEEE Conf. on Decision and Control (CDC) (1991) 437-442.

[25] H.J. Sussmann, Lie brackets and local controllability: a sufficient condition for scalar-input systems. SIAM J. Contr. Opt. 21 (1983) 686-713.

[26] H.J. Sussmann, A general theorem on local controllability. SIAM J. Contr. Opt. 25 (1987) 158-194. 